\title{
AIAA 99-3360
}

A Pseudo-Temporal Multi-Grid

Relaxation Scheme for Solving the

Parabolized Navier-Stokes

\section{Equations}

J. A. White

NASA Langley Research Center

Hampton, VA 23681

J. H. Morrison

Analytical Services and Materials, Inc.

107 Research Drive

Hampton, VA 23666

\section{4th AIAA Computational Fluid Dynamics Conference June 28-July 1, 1999/Norfolk, VA}




\title{
A Pseudo-Temporal Multi-Grid Relaxation Scheme for Solving the Parabolized Navier-Stokes Equations
}

\author{
J. A. White* \\ NASA Langley Research Center, Hampton, VA 23681 \\ J. H. Morrison ${ }^{\dagger}$ \\ Analytical Services and Materials, Inc., Hampton, VA 23666
}

\begin{abstract}
A multi-grid, flux-difference-split, finite-volume code, VULCAN, is presented for solving the elliptic and parabolized form of the equations governing three-dimensional, turbulent, calorically perfect and non-equilibrium chemically reacting flows. The space marching algorithms developed to improve convergence rate and or reduce computational cost are emphasized. The algorithms presented are extensions to the class of implicit pseudo-time iterative, upwind space-marching schemes. A full approximate storage, full multi-grid scheme is also described which is used to accelerate the convergence of a Gauss-Seidel relaxation method. The multi-grid algorithm is shown to significantly improve convergence on high aspect ratio grids.
\end{abstract}

\section{Introduction}

Scramjet engines and nozzles employed by hypersonic vehicles operate in a viscous supersonic/hypersonic flow regime. Such conditions allow the use of space-marching solution algorithms without adversely affecting the fidelity of the calculation when the flow is steady and unseparated in the marching direction. Numerous algorithms have been developed for solving the Euler, Navier-Stokes, and Parabolized Navier-Stokes (PNS) equations (e.g. Refs. 1-7) for a thermally and calorically perfect gas. Extensions of space marching algorithms have also been made to handle both equilibrium and non-equilibrium effects (e.g. Refs. 8-15). In addition, the PNS equations can be solved much more efficiently than the Navier-Stokes equations, which makes the PNS equations very attractive for use in design and optimization methods. ${ }^{16}$

Space marching methods can be broken down algorithmically into two groups, steady-state equation solvers and pseudo-time iterative solvers. Steadystate equation solvers, as the name implies, solve the steady-state form of the Euler and PNS equations. Explicit $3,5,10,17$ and implicit ${ }^{4,11,14}$ steady-state space-marching schemes have been used with considerable success. Pseudo-time iterative space-marching

*Aerospace Engineer, Hypersonic Airbreathing Propulsion Branch, Aero- and Gas-Dynamics Division, Research Technology Group.

${ }^{\dagger}$ Senior Research Scientist, Senior Member AIAA.

Copyright (C) 1999 by the American Institute of Aeronautics and Astronautics. Inc. No copyright is asserted in the United States under Title 17, U.S. Code. The U.S. Government has a royaltyfree license to exercise all rights under the copyright claimed herein for Governmental Purposes. All other rights are reserved by the copyright owner. schemes that solve the unsteady form of the Euler and PNS equations ${ }^{2,18,19}$ have also been employed using relaxation schemes that iterate at each spatial step in pseudo-time to reach a steady-state solution.

A large number of the problems of interest can include subsonic and/or separated flow regions, which requires that the space marching algorithm be implemented within the broader context of a code capable of solving the Navier-Stokes equations. VULCAN (Viscous Upwind aLgorithm for Complex flow ANalysis) is a cell-centered finite-volume, structured grid, multi-block code which solves the equations governing inviscid and viscous flow of a calorically perfect gas or of an arbitrary mixture of thermally perfect gases undergoing non-equilibrium chemical reactions. VULCAN allows the flow domain to be decomposed into regions in which the most suitable algorithm (elliptic or marching) can be utilized. The inviscid fluxes are computed using the MUSCL scheme ${ }^{20}$ with either the approximate Riemann solver of Roe ${ }^{21}$ or the low dissipation flux splitting scheme of Edwards. ${ }^{22}$ The viscous fluxes can be evaluated either with or without cross derivative contributions. VULCAN solves elliptic flows by marching the unsteady form of governing equations in time. Hyperbolic flows (e.g. the Euler equations in supersonic flow) and parabolic flows (e.g. the parabolized Navier-Stokes equations) are solved by space marching while iterating the unsteady equations in pseudo-time to a steady state solution. The pseudo-time iterative approach was chosen because it allows the time marching solution algorithms and convergence acceleration techniques developed to solve the elliptic Euler equation and FNS equations 
to be used to reduce the computational cost of the space marching scheme. The explicit PNS algorithm described in a previous paper, ${ }^{23}$ while extremely efficient for inviscid and moderate Reynolds number flows, was found to be too computationally expensive when solving high Reynolds number flows due to the large number of space marching steps required by stability constraints. The code structure of VULCAN was designed to allow the elliptic and space marching schemes to share numerical algorithms developed to solve and/or accelerate the convergence of the governing equations to steady state. The code has incorporated within it four time marching schemes: a multi-stage Runge-Kutta scheme, a diagonalized approximate factorization scheme, a block approximate factorization scheme, and a diagonally dominant alternating direction implicit scheme with a factorization error correction sub-iteration. Each of these schemes can be used as the smoothing algorithm in a full approximate storage (FAS) full multi-grid (FMG) scheme and thereby accelerate the convergence to steady state.

\section{Governing Equations}

The unsteady three-dimensional form of the Favre averaged Navier-Stokes and species transport equations can be written in nonorthogonal curvilinear form as

$$
\mathbf{Q}_{t}+\left(\mathbf{E}-\mathbf{E}_{v}\right)_{\xi}+\left(\mathbf{F}-\mathbf{F}_{v}\right)_{\eta}+\left(\mathbf{G}-\mathbf{G}_{v}\right)_{\zeta}=\mathbf{S}
$$

where the subscripts $\xi, \eta$, and $\zeta$ denote partial differentiation with respect to the nonorthogonal curvilinear coordinates and the subscript $t$ denotes partial differentiation with respect to time. The contravariant components of the curvilinear nonorthogonal coordinate system are

$$
\xi=\xi(x, y, z) ; \eta=\eta(x, y, z) ; \zeta=\zeta(x, y, z)
$$

and

$$
J=\left|\frac{\partial(\xi, \eta, \zeta)}{\partial(x, y, z)}\right|
$$

where $\xi_{\alpha}, \eta_{\alpha}$, and $\xi_{\alpha}(\alpha=\mathrm{x}, \mathrm{y}$, or $\mathrm{z})$ are the components of the cell face area normal vector, while $\hat{\xi}_{\alpha}, \hat{\xi}_{\alpha}$, and $\hat{\xi}_{\alpha}$ are the components of the cell face unit normal.

The $\mathbf{Q}, \mathbf{E}, \mathbf{F}, \mathbf{G}$, and $\mathbf{S}$ vectors are defined using Favre averaged variables (indicated as $\tilde{\phi}$ ) and Reynolds averaged variables (indicated as $\bar{\phi}$ ) as

$$
\mathbf{Q}=\frac{1}{J}\left[\begin{array}{c}
\bar{\rho} \tilde{f}_{i} \\
\vdots \\
\bar{\rho} \tilde{f}_{N_{c s}} \\
\bar{\rho} \tilde{u} \\
\bar{\rho} \tilde{v} \\
\bar{\rho} \tilde{w} \\
\bar{\rho} \tilde{E} \\
\bar{\rho} \tilde{k} \\
\bar{\rho} \tilde{l} \\
\bar{\rho} \tilde{g} \\
\bar{\rho} \tilde{\mathcal{F}}
\end{array}\right] \quad \mathbf{S}=\left[\begin{array}{c}
\overline{\dot{\omega}_{i}} \\
\vdots \\
\overline{\dot{\omega}_{N_{c s}}} \\
0 \\
0 \\
0 \\
0 \\
S_{\tilde{k}} \\
S_{\tilde{l}} \\
S_{\tilde{g}} \\
S_{\tilde{\mathcal{F}}}
\end{array}\right]
$$

$$
\mathbf{E}=\frac{|\nabla \xi|}{J}\left[\begin{array}{c}
\bar{\rho} \tilde{f}_{i} U \\
\vdots \\
\bar{\rho} \tilde{f}_{N_{c s}} U \\
\bar{\rho} \tilde{u} U+\hat{\xi}_{x} \bar{p} \\
\bar{\rho} \tilde{v} U+\hat{\xi}_{y} \bar{p} \\
\bar{\rho} \tilde{w} U+\hat{\xi}_{z} \bar{p} \\
\bar{\rho} \tilde{H} U \\
\bar{\rho} \tilde{k} U \\
\bar{\rho} \tilde{l} U \\
\bar{\rho} \tilde{g} U \\
\bar{\rho} \tilde{\mathcal{F}} U
\end{array}\right]
$$

$$
\mathbf{F}=\frac{|\nabla \eta|}{J}\left[\begin{array}{c}
\bar{\rho} \tilde{f}_{i} V \\
\vdots \\
\bar{\rho} \tilde{f}_{N_{c s}} V \\
\bar{\rho} \tilde{u} V+\hat{\eta}_{x} \bar{p} \\
\bar{\rho} \tilde{v} V+\hat{\eta}_{y} \bar{p} \\
\bar{\rho} \tilde{w} V+\hat{\eta}_{z} \bar{p} \\
\bar{\rho} \tilde{H} V \\
\bar{\rho} \tilde{k} V \\
\bar{\rho} \tilde{l} V \\
\bar{\rho} \tilde{g} V \\
\bar{\rho} \tilde{\mathcal{F} V}
\end{array}\right]
$$

$$
\mathbf{G}=\frac{|\nabla \zeta|}{J}\left[\begin{array}{c}
\bar{\rho} \tilde{f}_{i} W \\
\vdots \\
\bar{\rho} \tilde{f}_{N_{c s}} W \\
\bar{\rho} \tilde{u} W+\hat{\zeta}_{x} \bar{p} \\
\bar{\rho} \tilde{v} W+\hat{\zeta}_{y} \bar{p} \\
\bar{\rho} \tilde{w} W+\hat{\zeta}_{z} \bar{p} \\
\bar{\rho} \tilde{H} W \\
\bar{\rho} \tilde{k} W \\
\bar{\rho} \tilde{l} W \\
\bar{\rho} \tilde{g} W \\
\bar{\rho} \tilde{\mathcal{F}} W
\end{array}\right]
$$

where $\bar{\rho}$ is the mixture density; $\tilde{f}_{i}$ is the mass fraction and $\overline{\dot{\omega}_{i}}$ is the species production rate of the $i^{t h}$ chemical species; $N_{c s}$ is the total number of chemical species; $\bar{p}$ is the static pressure; $\tilde{u}, \tilde{v}$ and $\tilde{w}$ are the Cartesian 
velocity components; and $\tilde{E}$ and $\tilde{H}$ are the total energy and total enthalpy respectively. The variables $\tilde{k}$ and $\tilde{l}$ are the turbulent kinetic energy and a turbulent length scale equation variable (either $\tilde{\omega}$ or $\tilde{\epsilon}$ ). The turbulence chemistry interaction variables $\tilde{g}$ and $\tilde{\mathcal{F}}$ are the energy variance $e^{\|} e^{\prime \prime}$, and the sum of the species variances $\sum_{i=1}^{N_{c s}} f_{i}^{\prime \prime} f_{i}^{\prime \prime}$. The contravariant velocities are defined as

$$
\begin{aligned}
U & =\hat{\xi}_{x} \tilde{u}+\hat{\xi}_{y} \tilde{v}+\hat{\xi}_{z} \tilde{w} \\
V & =\hat{\eta}_{x} \tilde{u}+\hat{\eta}_{y} \tilde{v}+\hat{\eta}_{z} \tilde{w} \\
W & =\hat{\zeta}_{x} \tilde{u}+\hat{\zeta}_{y} \tilde{v}+\hat{\zeta}_{z} \tilde{w}
\end{aligned}
$$

The viscous flux vectors $\mathbf{E}_{v}, \mathbf{F}_{v}$, and $\mathbf{G}_{v}$ are written as

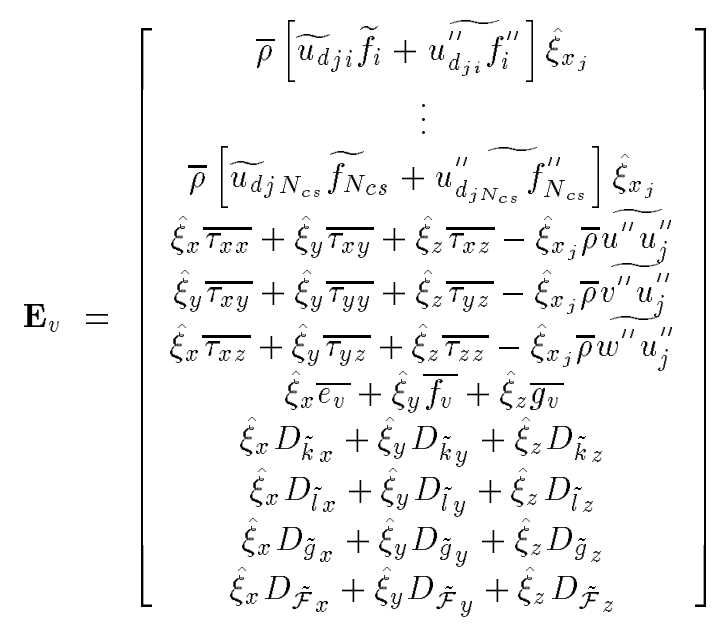

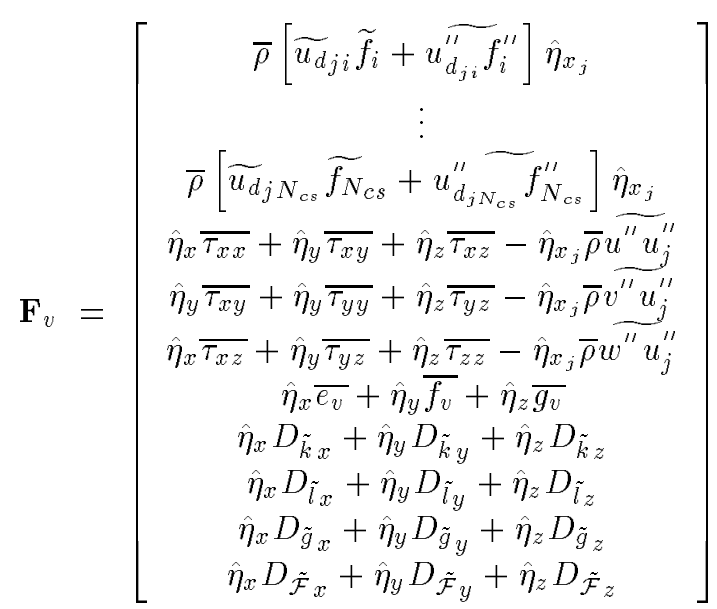

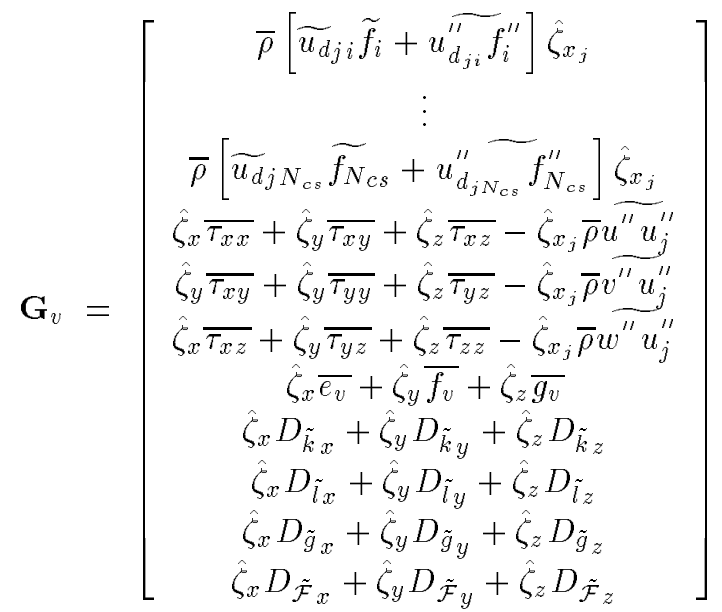

The components of the species molecular diffusion velocity of the $i^{t h}$ specie in the $j^{t h}$ direction, $\widetilde{u}_{d j i} \tilde{f}_{i}$, are expressed using a Fickian model and their turbulent counterparts, $u_{d_{j i}}^{\prime \prime} f_{i}^{\prime \prime}$, are closed using a gradient diffusion model as

$$
\bar{\rho}\left[\widetilde{u}_{d j i} \tilde{f}_{i}+\widetilde{u}_{d_{j i}}^{u_{i}^{\prime \prime}}\right]=-\left(\frac{\mu}{S c}+\frac{\mu_{t}}{S c_{t}}\right) \frac{\partial \tilde{f}_{i}}{\partial x_{j}}
$$

where $\mu$ and $\mu_{t}$ are the molecular and turbulent viscosity and $S c$ and $S c_{t}$ are the laminar and turbulent Schmidt numbers. The Cartesian components of the viscous energy flux are

$$
\begin{aligned}
& \overline{e_{v}}=\tilde{u} \overline{\tau_{x x}}+\tilde{v} \overline{\tau_{x y}}+\tilde{w} \overline{\tau_{x z}}-\overline{q_{x}}+D_{\tilde{k} x} \\
& \overline{f_{v}}=\tilde{u} \overline{\tau_{x y}}+\tilde{v} \overline{\tau_{y y}}+\tilde{w} \overline{\tau_{y z}}-\overline{q_{y}}+D_{\tilde{k} y} \\
& \overline{g_{v}}=\tilde{u} \overline{\tau_{x z}}+\tilde{v} \overline{\tau_{y z}}+\tilde{w} \overline{\tau_{z z}}-\overline{q_{z}}+D_{\tilde{k} z}
\end{aligned}
$$

where $\sigma_{k}$ is the turbulent Prandtl number for the diffusion of turbulent kinetic energy. The Cartesian viscous stresses are defined as

$$
\begin{aligned}
& \overline{\tau_{x x}}=\mu\left[2 \frac{\partial \tilde{u}}{\partial x}-\frac{2}{3}\left(\frac{\partial \tilde{u}}{\partial x}+\frac{\partial \tilde{v}}{\partial y}+\frac{\partial \tilde{w}}{\partial z}\right)\right] \\
& \overline{\tau_{y y}}=\mu\left[2 \frac{\partial \tilde{v}}{\partial y}-\frac{2}{3}\left(\frac{\partial \tilde{u}}{\partial x}+\frac{\partial \tilde{v}}{\partial y}+\frac{\partial \tilde{w}}{\partial z}\right)\right] \\
& \overline{\tau_{z z}}=\mu\left[2 \frac{\partial \tilde{w}}{\partial z}-\frac{2}{3}\left(\frac{\partial \tilde{u}}{\partial x}+\frac{\partial \tilde{v}}{\partial y}+\frac{\partial \tilde{w}}{\partial z}\right)\right] \\
& \overline{\tau_{x y}}=\mu\left(\frac{\partial \tilde{u}}{\partial y}+\frac{\partial \tilde{v}}{\partial x}\right) \\
& \overline{\tau_{x z}}=\mu\left(\frac{\partial \tilde{u}}{\partial z}+\frac{\partial \tilde{w}}{\partial x}\right) \\
& \overline{\tau_{y z}}=\mu\left(\frac{\partial \tilde{v}}{\partial z}+\frac{\partial \tilde{w}}{\partial y}\right)
\end{aligned}
$$

and the total heat fluxes are given as

$$
\begin{aligned}
& \overline{q_{x}}=-\left(\lambda+\frac{\mu_{t}}{P r_{t}}\right) \frac{\partial \tilde{T}}{\partial x}-\bar{\rho}\left(\frac{\mu}{S c}+\frac{\mu_{t}}{S c_{t}}\right) \sum_{i=1}^{N_{c s}} \tilde{h}_{i} \tilde{f}_{i x} \\
& \overline{q_{y}}=-\left(\lambda+\frac{\mu_{t}}{P r_{t}}\right) \frac{\partial \tilde{T}}{\partial y}-\bar{\rho}\left(\frac{\mu}{S c}+\frac{\mu_{t}}{S c_{t}}\right) \sum_{i=1}^{N_{c s}} \tilde{h}_{i} \tilde{f}_{i y}
\end{aligned}
$$


$\overline{q_{z}}=-\left(\lambda+\frac{\mu_{t}}{P r_{t}}\right) \frac{\partial \tilde{T}}{\partial z}-\bar{\rho}\left(\frac{\mu}{S c}+\frac{\mu_{t}}{S c_{t}}\right) \sum_{i=1}^{N_{c s}} \tilde{h}_{i} \tilde{f}_{i_{z}}$

where $\lambda$ is the gas mixture molecular thermal conductivity and $P r_{t}$ is the turbulent Prandtl number. The turbulence modeling equation diffusion terms $D_{\tilde{\phi}_{x}}$, $D_{\tilde{\phi}_{y}}$, and $D_{\tilde{\phi}_{z}}$, where $\phi$ is either $\tilde{k}, \tilde{\epsilon}, \tilde{\omega}, \tilde{g}$, or $\tilde{\mathcal{F}}$, are modeled as

$$
\begin{aligned}
& D_{\tilde{\phi}_{x}}=\left(\frac{\mu}{P r_{l \phi}}+\frac{\mu_{t}}{P r_{t \phi}}\right) \tilde{\phi}_{x} \\
& D_{\tilde{\phi}_{y}}=\left(\frac{\mu}{P r_{l \phi}}+\frac{\mu_{t}}{P r_{t \phi}}\right) \tilde{\phi}_{y} \\
& D_{\tilde{\phi}_{z}}=\left(\frac{\mu}{P r_{l \phi}}+\frac{\mu_{t}}{P r_{t \phi}}\right) \tilde{\phi}_{z}
\end{aligned}
$$

\section{Turbulence Models}

The Reynolds stress term $\left(\bar{\rho} u_{i}{ }^{\prime \prime} u_{j}{ }^{\prime \prime}\right)$ model is dependent on the type of turbulence model selected. Currently, only two equation models are available in VULCAN. However, the two equation model implementation allows flexibility when modeling the Reynolds stresses. If one of the eddy viscosity based two equation turbulence models is selected, the Boussinesq approximation is made where the Reynolds stresses are modeled as

$-\bar{\rho} u_{i}{ }^{\prime \prime} u_{j}{ }^{\prime \prime}=\mu_{t}\left[\left(\frac{\partial \tilde{u}_{i}}{\partial x_{j}}+\frac{\partial \tilde{u_{j}}}{\partial x_{i}}\right)-\frac{2}{3} \frac{\partial \tilde{u_{k}}}{\partial x_{k}} \delta_{i j}\right]-\frac{2}{3} \bar{\rho} \tilde{k} \delta_{i j}$

If an explicit algebraic Reynolds stress two equation turbulence model is selected, then the Reynolds stresses are modeled as

$$
\begin{aligned}
& -\bar{\rho} u_{i}{ }^{\prime \prime} u_{j}{ }^{\prime \prime}=-\frac{2}{3} \bar{\rho} \tilde{k} \delta_{i j}+2 \mu_{t}^{*}\left(S_{i j}-\frac{1}{3} S_{k k} \delta_{i j}\right) \\
& +2 \mu_{t}^{* *}\left[\frac{\alpha_{4}}{\tilde{\omega}}\left(S_{i k} W_{k j}+S_{j k} W_{k i}\right)\right] \\
& -2 \mu_{t}^{* *}\left[\frac{\alpha_{5}}{\tilde{\omega}}\left(S_{i k} S_{k j}-\frac{1}{3} S_{k l} S_{k l} \delta_{i j}\right)\right]
\end{aligned}
$$

where

$$
\begin{aligned}
S_{i j} & =\frac{1}{2}\left(\frac{\partial \tilde{u}_{i}}{\partial x_{j}}+\frac{\partial \tilde{u}_{j}}{\partial x_{i}}\right) \\
W_{i j} & =\frac{1}{2}\left(\frac{\partial \tilde{u}_{i}}{\partial x_{j}}-\frac{\partial \tilde{u}_{j}}{\partial x_{i}}\right)
\end{aligned}
$$

and $\tilde{\omega}=\tilde{\epsilon} / \tilde{k}$. The definitions for $\mu_{t}^{*}, \mu_{t}^{* *}, \alpha_{4}$, and $\alpha_{5}$ can be found in Abid et al. ${ }^{25}$

The two equation turbulence models in VULCAN can be categorized as $\tilde{k}-\tilde{\epsilon}$ based models, Wilcox's ${ }^{28}$ $\tilde{k}-\tilde{\omega}$ based models and Menter's ${ }^{24} \tilde{k}-\tilde{\omega}$ models. The $\tilde{k}-\tilde{\epsilon}$ based models can be solved with either the Boussinesq model or an explicit algebraic Reynolds stress model. ${ }^{25-27}$ The Wilcox $\tilde{k}-\tilde{\omega}$ based models are available in three forms; a high Reynolds number model, ${ }^{28}$ a low Reynolds number model, ${ }^{28}$ and an explicit algebraic Reynolds stress model. ${ }^{27}$ The $\tilde{k}-\tilde{\omega}$ models of
Menter ${ }^{24}$ are available in two forms; the baseline model and the Shear Stress Transport (SST) model. A correction to each model for compressibility effects has also been implemented using the compressible dissipation model of Sarkar et al. ${ }^{29}$ for the $\tilde{k}-\tilde{\epsilon}$ based models and the compressibility model suggested by Wilcox, ${ }^{28}$ which incorporates Sarkar's model as well as Zeman's lagging function, ${ }^{30}$ for all of the $\tilde{k}-\tilde{\omega}$ based models (including Menter's).

\section{Near Wall Turbulence Models}

The near wall behavior of the $\tilde{k}-\tilde{\epsilon}$ two equation models is controlled through the introduction of the low Reynolds number modifications of Abid. ${ }^{31}$ The near wall behavior of the $\tilde{k}-\tilde{\omega}$ two equation models is treated in either a high Reynolds number manner by integrating the high Reynolds number form of the equations to the wall without any special wall treatment or by integrating the low Reynolds form of the Wilcox $\tilde{k}-\tilde{\omega}$ two equation model ${ }^{28}$ to the wall.

If integration to the wall is not feasible for a given problem, the wall matching function approach of Wilcox ${ }^{28}$ can be used. This approach models the wall shear stress and heat transfer by enforcing a compressible law of the wall which includes additional terms that model streamwise pressure gradients. Wilcox ${ }^{32}$ showed that inclusion of the pressure gradient terms in the wall matching function dramatically improved predictions of shear stress. The wall matching function has been successfully used on grids where the $y^{+}$ $\left(=\sqrt{\tau_{w}} / \rho ; \tau_{w}\right.$ is the wall shear stress) varied between 0.5 and 150.0 and produced wall skin friction and heat transfer predictions that agreed well with results obtained on grids that were integrated to the wall for $y^{+}<1.0$. This is a very useful characteristic which significantly reduces flow solution sensitivity to the grid and greatly reduces the required amount of a priori knowledge about the range of $y^{+}$when generating the computational grid.

\section{Pressure and Thermodynamic Models}

VULCAN was written to model the working gas either as a single component calorically perfect gas or as an arbitrary mixture of thermally perfect gases. For a single component calorically perfect gas, the solution vector of conserved variables in Eq. (4) is contracted to contain only a single continuity equation $\bar{\rho}$ (with $\overline{\dot{\omega}}=0$ ), three momentum equations $\bar{\rho} \tilde{u}, \bar{\rho} \tilde{v}$, and $\bar{\rho} \tilde{w}$, the energy equation $\bar{\rho} \tilde{E}$ and the turbulence equations for $\bar{\rho} \tilde{k}$ and $\bar{\rho} \tilde{l}$. The pressure, static enthalpy, total enthalpy, and total energy for a single component calorically perfect gas are

$$
\begin{gathered}
\bar{p}=\bar{\rho} R \tilde{T} \\
\tilde{h}=\left(\frac{\gamma R}{\gamma-1}\right) \tilde{T} \\
\tilde{H}=\tilde{h}+\frac{1}{2}\left(\tilde{u}^{2}+\tilde{v}^{2}+\tilde{w}^{2}\right)+\tilde{k}
\end{gathered}
$$




$$
\tilde{E}=\tilde{H}-\frac{\bar{p}}{\bar{\rho}}
$$

where $R$ is the gas constant and $\gamma$ is the ratio of specific heats.

The pressure, species enthalpy, static enthalpy, total enthalpy and total energy for an arbitrary mixture of thermally perfect gases are modeled as

$$
\begin{gathered}
\bar{p}=\bar{\rho} \mathcal{R}_{u} \tilde{T} \sum_{i=1}^{N_{c s}} \frac{\tilde{f}_{i}}{\mathcal{M}_{i}} \\
\tilde{h_{i}}=\tilde{h_{i}^{o}}+\int_{T_{o}}^{\tilde{T}} C_{p_{i}} d T \\
\tilde{h}=\sum_{i=1}^{N_{c s}} \tilde{h_{i}} \tilde{f}_{i}
\end{gathered}
$$

where $\mathcal{R}_{u}$ is the universal gas constant, $\mathcal{M}_{i}$ and $R_{i}$ $\left(=\mathcal{R}_{u} / \mathcal{M}_{i}\right)$ are the molecular weight and gas constant of the $i^{t h}$ species, respectively, and $h_{i}^{\circ}$ is the reference enthalpy at a reference temperature, $T_{o}$, of $i^{t h}$ chemical species. The specific heat at constant pressure, enthalpy, and Gibbs free energy for each chemical species are modeled with a polynomial of static temperature. For example, the $C_{p_{i}}$ polynomial is modeled as either ${ }^{33}$

$$
\frac{C_{p_{i}}}{R_{i}}=A_{i}+B_{i} \tilde{T}+C_{i} \tilde{T}^{2}+D_{i} \tilde{T}^{3}+E_{i} \tilde{T}^{4}
$$

or $\operatorname{as}^{34}$

$$
\frac{C_{p_{i}}}{R_{i}}=\frac{A_{i}}{\tilde{T}^{2}}+\frac{B_{i}}{\tilde{T}}+C_{i}+D_{i} \tilde{T}+E_{i} \tilde{T}^{2}+F_{i} \tilde{T}^{3}+G_{i} \tilde{T}^{4}
$$

The coefficients $A_{i}, B_{i}, C_{i}, D_{i}, E_{i}, F_{i}$, and $G_{i}$ are read from thermodynamic curve fit data base files compiled for each polynomial from the data in McBride et al. ${ }^{33,34}$

\section{Molecular Transport Models}

The molecular viscosity, $\mu$, of a single-component gas is modeled using Sutherland's formula where

$$
\mu=\mu_{0}\left(\frac{\tilde{T}}{T_{0}}\right)^{\frac{3}{2}} \frac{T_{0}+S}{\tilde{T}+S}
$$

where $\mu_{o}, T_{o}$, and $S$ are constants that are specified for the gas of interest. The molecular viscosity, $\mu_{i}$, of each component of a multicomponent gas is modeled using Sutherland's formula

$$
\mu_{i}=\mu_{o, i}\left(\frac{\tilde{T}}{T_{o, i}}\right)^{\frac{3}{2}} \frac{T_{o, i}+S_{i}}{\tilde{T}+S_{i}}
$$

where $\mu_{o, i}, T_{o, i}$, and $S_{i}$ are constants that are specific to the chemical species of interest and are available as part of the thermodynamics data base supplied with
VULCAN. Wilke's formula ${ }^{35}$ is then applied to model the mixture molecular viscosity as

$$
\mu=\sum_{i=1}^{N_{c s}} \frac{\chi_{i} \mu_{i}}{\sum_{j=1}^{N_{c s}} \chi_{j} \phi_{i j}}
$$

where

$$
\phi_{i j}=\frac{\left[1+\left(\frac{\mu_{i}}{\mu_{j}}\right)^{\frac{1}{2}}\left(\frac{\mathcal{M}_{j}}{\mathcal{M}_{i}}\right)^{\frac{1}{4}}\right]^{2}}{\sqrt{8}\left(1+\frac{\mathcal{M}_{i}}{\mathcal{M}_{j}}\right)^{\frac{1}{2}}}
$$

and $\chi_{i}$ is the $i_{t h}$ chemical species mole fraction.

The molecular thermal conductivity, $\lambda$, of a singlecomponent gas is modeled using the Prandtl number such that

$$
\lambda=\frac{C_{p} \mu}{P r}
$$

The molecular thermal conductivity of a multicomponent gas is modeled using either using Eqs. $(32,33,34)$ or by using Sutherland's formula for the chemical species molecular thermal conductivity $\lambda_{i}$ such that

$$
\lambda_{i}=\lambda_{o, i}\left(\frac{\tilde{T}}{\mathcal{T}_{o, i}}\right)^{\frac{3}{2}} \frac{\mathcal{T}_{o, i}+\mathcal{S}_{i}}{\tilde{T}+\mathcal{S}_{i}}
$$

where $\lambda_{o, i}, \mathcal{T}_{o, i}$, and $\mathcal{S}_{i}$ are constants that are specific to the chemical species of interest and are available as part of the thermodynamics data base supplied with VULCAN. Wassiljewa's formula ${ }^{36}$ is then applied to model the mixture molecular viscosity

$$
\lambda=\sum_{i=1}^{N_{c s}} \frac{\chi_{i} \lambda_{i}}{\sum_{j=1}^{N_{c s}} \chi_{j} \Phi_{i j}}
$$

where

$$
\Phi_{i j}=\frac{\left[1+\left(\frac{\lambda_{i}}{\lambda_{j}}\right)^{\frac{1}{2}}\left(\frac{\mathcal{M}_{j}}{\mathcal{M}_{i}}\right)^{\frac{1}{4}}\right]^{2}}{\sqrt{8}\left(1+\frac{\mathcal{M}_{i}}{\mathcal{M}_{j}}\right)^{\frac{1}{2}}}
$$

\section{Chemical Source Term Model}

The chemical source term, $\overline{\dot{\omega}_{i}}$, represents the mean of the net rate of production of the $i^{\text {th }}$ species in each chemical reaction. This source term can be modeled either with or without turbulence/chemistry interaction effects. The chemical source term, without turbulence/chemistry interaction effects, is modeled in the manner of ${ }^{39}$ such that

$\overline{\dot{\omega}_{i}}=\mathcal{M}_{i} \sum_{j=1}^{N_{c r}}\left(\nu_{j i}^{\prime \prime}-\nu_{j i}^{\prime}\right)\left[\mathcal{K}_{f_{j}} \prod_{m=1}^{N_{c s}} \overline{\mathcal{C}}_{m}{ }^{\nu_{j m}^{\prime}}-\mathcal{K}_{b_{j}} \prod_{n=1}^{N_{c s}} \overline{\mathcal{C}}_{n}{ }^{\nu_{j n}^{\prime \prime}}\right]$

Eq. (38) is the production rate of the ith species as determined by the law of mass action where $C_{m}$ and $C_{n}$ are the participating species concentrations, $\nu_{j m}^{\prime}$ and 
$\nu_{j n}^{\prime \prime}$ their stoichiometric coefficients, and $\mathcal{K}_{f_{j}}$ and $\mathcal{K}_{b_{j}}$ the reaction rate coefficients of the forward and backward reactions. In addition, the constraint imposed by mass conservation exists such that $\sum_{i=1}^{N_{c s}} \overline{\dot{\omega}_{i}}=0$. The forward and backward reaction rate coefficients are calculated based on the Arrhenius law and the equilibrium rate coefficient, which are defined as

$$
\begin{gathered}
\mathcal{K}_{f_{j}}=\mathcal{A}_{j} \tilde{T}^{\mathcal{B}_{j}} \exp \left(\frac{-\mathcal{E}_{a j}}{\mathcal{R}_{u} \tilde{T}}\right) \\
\mathcal{K}_{b_{j}}=\frac{\mathcal{K}_{f_{j}}}{\mathcal{K}_{e q_{j}}}
\end{gathered}
$$

The constants in the Arrhenius law, $\mathcal{A}_{j}, \mathcal{B}_{j}$, and $\mathcal{E}_{a j}$, for a hydrogen-air reaction system and a complete description of the method used to obtain the equilibrium rate coefficient can be found in Drummond and Hussaini $^{37}$ or Carpenter. ${ }^{40}$

The chemical source term model which includes the effects of turbulence/chemistry interaction uses assumed probability density functions (PDF) to compute the mean species production rate. In this approach, the law of mass action is written as

$\overline{\dot{\omega}_{i}}=\mathcal{M}_{i} \sum_{j=1}^{N_{c r}}\left(\nu_{j i}^{\prime \prime}-\nu_{j i}^{\prime}\right)\left[\overline{\mathcal{K}_{f_{j}}} \overline{\prod_{m=1}^{N_{c s}} \mathcal{C}_{m}^{\nu_{j m}^{\prime}}}-\overline{\mathcal{K}_{b_{j}}} \prod_{n=1}^{\overline{N_{c s}} \mathcal{C}_{n}^{\nu^{\prime \prime}{ }^{\prime \prime}}}\right]$

The mean reaction rate coefficients, $\overline{\mathcal{K}}_{f_{j}}$ and $\overline{\mathcal{K}}_{b_{j}}$, are computed using a truncated Taylor series expansion of the rate coefficient equations with respect to the mean and fluctuating static temperature, where the static temperature fluctuation $T^{\prime \prime}$ is obtained from the solution of the energy variance, $e^{\pi} e^{\prime \prime}$, transport equation. The higher order moments of $T^{\prime \prime}$ introduced by the Taylor series expansion are computed from either an assumed Gaussian or $\beta$ PDF as described in Gaffney et al. ${ }^{41,43}$ The mean forward and backward species production terms $\overline{\prod_{m=1}^{N_{c s}} \mathcal{C}_{m}^{\nu_{j m}^{\prime}}}$ and $\overline{\prod_{n=1}^{N_{c s}} \mathcal{C}_{n}^{\nu^{\prime \prime}}}$ are computed from a multivariate $\beta$ PDF where the sum of the species variance $\sum_{i=1}^{N_{c s}} \widehat{f_{i}^{\prime \prime} f_{i}^{\prime \prime}}$ transport equation is solved and the variance is used in the manner described in Girimaji ${ }^{42}$ and Gaffney et al. ${ }^{43}$

\section{Spatial Discretization}

The governing equations, Eq. (1), are discretized with a cell centered, finite volume representation. The convection terms are treated in a second order accurate manner with the primitive variable extrapolation/interpolation scheme (MUSCL) of van Leer. ${ }^{20}$ Discontinuities such as shocks are captured by employing flux limiters or by variants on the MUSCL scheme which employ differentiable limiters. ${ }^{44,45}$ The numerical flux at the cell interface may be constructed using a flux-difference-split scheme where the flux construction is defined as

$$
\mathbf{E}_{I}=\frac{1}{2}\left(\mathbf{E}^{L}+\mathbf{E}^{R}\right)-\frac{1}{2}|\hat{\tilde{\mathbf{A}}}|\left(\mathbf{Q}^{R}-\mathbf{Q}^{L}\right)
$$

where $I$ represents the cell interface, $L$ and $R$ represent the left and right states, and $|\hat{\tilde{A}}|$ is due to Roe's approximate Riemann solver. ${ }^{21}$ A low dissipation flux splitting scheme based on the method of Edwards ${ }^{22}$ that addresses some of the entropy violation properties of Roe's scheme is also implemented. The low dissipation flux split flux construction is defined as

$\mathbf{E}_{I}=a_{\frac{1}{2}}\left[\rho_{L} C^{+} \mathbf{E}_{L}^{c}-\rho_{R} C^{-} \mathbf{E}_{R}^{c}\right]+\mathbf{E}^{p}\left[D_{L}^{+} p_{L}+D_{R}^{-} p_{R}\right]$

where $\mathbf{E}^{c}$ is the convection contribution to the flux and $\mathbf{E}^{p}$ is the pressure contribution to the flux. The parameters $C^{ \pm}$and $D^{ \pm}$(see Ref. 22) are functions of the left and right contravariant Mach number and $a_{\frac{1}{2}}=\left(a_{L}+a_{R}\right) / 2$ with $a$ the frozen speed of sound. In both flux construction methods, the MUSCL scheme is used to reconstruct values of the primitive variables at the left and right states. The primitive variables used are the species mass fraction $\tilde{f}_{i}$, the density $\bar{\rho}$, the Cartesian velocity components $\tilde{u}, \tilde{v}$, and $\tilde{w}$, the static pressure $\bar{p}$, and the turbulence variables $\tilde{k}, \tilde{l}, \tilde{g}$, and $\tilde{\mathcal{F}}$.

The viscous fluxes are discretized using a finitevolume representation of a central difference operator $^{46}$ which can be configured to evaluate the cell interface gradients using either an approximate gradient construction where the cross-derivative terms are neglected or a full gradient construction in which all components of the derivative are included. ${ }^{46}$

\section{Elliptic Solution Procedures}

The Navier-Stokes equations, Eq. (1), are hyperbolic in time and elliptic in space and, therefore, can be integrated in time to provide a steady state solution that is elliptic in space. VULCAN has several time integration procedures from which to choose, depending on the type of problem being solved. A temporally second order accurate low storage, explicit, multi-stage Runge-Kutta scheme ${ }^{47}$ can be used to integrate either to a steady state solution or in a time accurate manner. Alternately, due to the stiff nature of the governing equations and the expense involved in computing the chemical source terms, implicit time integration schemes can be selected to integrate the equations to steady state. An Euler implicit time integration scheme is written in delta form as:

$$
\left[\frac{\mathbf{I}}{J \Delta t}+\delta_{\xi} \mathbf{E}_{\mathbf{Q}}+\delta_{\eta} \mathbf{F}_{\mathbf{Q}}+\delta_{\zeta} \mathbf{G}_{\mathbf{Q}}-\mathbf{S}_{\mathbf{Q}}\right] \Delta \mathbf{Q}=-\mathbf{R}^{\mathbf{n}}
$$

where $\mathbf{E}_{\mathbf{Q}}=\frac{\partial \mathbf{E}}{\partial \mathbf{Q}}-\frac{\partial \mathbf{E}_{\mathbf{v}}}{\partial \mathbf{Q}}, \mathbf{F}_{\mathbf{Q}}=\frac{\partial \mathbf{F}}{\partial \mathbf{Q}}-\frac{\partial \mathbf{F}_{\mathbf{v}}}{\partial \mathbf{Q}}, \mathbf{G}_{\mathbf{Q}}=$ $\frac{\partial \mathbf{G}}{\partial \mathbf{Q}}-\frac{\partial \mathbf{G}_{\mathbf{v}}}{\partial \mathbf{Q}}, \mathbf{S}_{\mathbf{Q}}=\frac{\partial \mathbf{S}}{\partial \mathbf{Q}}, \Delta \mathbf{Q}=\mathbf{Q}^{n+1}-\mathbf{Q}^{n}$, and $R^{n}$ is the steady state residual at time $n$. The direct solution of Eq. (44) results in a large banded $N \times N$ system of equations (where $N$ is the number of equations) which must be inverted at each time step.

Solving Eq. (44) for even a medium sized threedimensional problem is impractical due to storage 
requirements. However, Eq. (44) may be factored to reduce the storage. Three approaches to the approximate factorization of Eq. (44) are currently implemented in VULCAN. The approach with the least factorization error and highest storage requirements is a planar Gauss-Seidel relaxation which uses a Diagonally Dominant Alternating Direction Implicit (DDADI) ${ }^{49}$ scheme to factor the cross stream plane. The forward sweep in $i$ of a nonlinear planar GaussSeidel relaxation of Eq. (44) may be written

$$
\begin{gathered}
{\left[\frac{\mathbf{I}}{J \Delta t}+\mathbf{B}_{\xi}+\mathbf{B}_{\eta}+\mathbf{B}_{\zeta}-\mathbf{S}_{\mathbf{Q}}\right] \Delta \mathbf{Q}_{i, j, k}+} \\
\mathbf{A}_{\eta} \Delta \mathbf{Q}_{i, j-1, k}+\mathbf{C}_{\eta} \Delta \mathbf{Q}_{i, j+1, k}+ \\
\mathbf{A}_{\zeta} \Delta \mathbf{Q}_{i, j, k-1}+\mathbf{C}_{\zeta} \Delta \mathbf{Q}_{i, j, k+1}= \\
-\mathbf{R}^{n}\left(\mathbf{Q}_{i-2, j, k}^{n+1}, \mathbf{Q}_{i-1, j, k}^{n+1}, \mathbf{Q}_{i, j, k}^{n}, \mathbf{Q}_{i+1, j, k}^{n}, \mathbf{Q}_{i+2, j, k}^{n}\right)
\end{gathered}
$$

and the backward sweep as

$$
\begin{gathered}
{\left[\frac{\mathbf{I}}{J \Delta t}+\mathbf{B}_{\xi}+\mathbf{B}_{\eta}+\mathbf{B}_{\zeta}-\mathbf{S}_{\mathbf{Q}}\right] \Delta \mathbf{Q}_{i, j, k}+} \\
\mathbf{A}_{\eta} \Delta \mathbf{Q}_{i, j-1, k}+\mathbf{C}_{\eta} \Delta \mathbf{Q}_{i, j+1, k}+ \\
\mathbf{A}_{\zeta} \Delta \mathbf{Q}_{i, j, k-1}+\mathbf{C}_{\zeta} \Delta \mathbf{Q}_{i, j, k+1}= \\
-\mathbf{R}^{n}\left(\mathbf{Q}_{i-2, j, k}^{n}, \mathbf{Q}_{i-1, j, k}^{n}, \mathbf{Q}_{i, j, k}^{n}, \mathbf{Q}_{i+1, j, k}^{n+1}, \mathbf{Q}_{i+2, j, k}^{n+1}\right)
\end{gathered}
$$

where $\mathbf{A}_{\alpha}, \mathbf{B}_{\alpha}$, and $\mathbf{C}_{\alpha}$ are block $N \times N$ matrices associated with replacing the $\delta_{\alpha}$ of Eq. (44) with a first order difference in the $\alpha=\xi, \eta$, and $\zeta$ directions. Each sweep is factored in the $\eta, \zeta$ plane as

$$
\begin{gathered}
{\left[\mathbf{M}+\theta\left(\mathbf{A}_{\eta}+\mathbf{C}_{\eta}\right)\right] \Delta \mathbf{Q}_{i, j, k}^{*}=-\mathbf{R}^{n}} \\
{\left[\mathbf{M}+\theta\left(\mathbf{A}_{\zeta}+\mathbf{C}_{\zeta}\right)\right] \Delta \mathbf{Q}_{i, j, k}=\mathbf{M} \Delta \mathbf{Q}_{i, j, k}^{*}} \\
\mathbf{Q}_{i, j, k}^{n+1}=\mathbf{Q}_{i, j, k}^{n}+\Delta \mathbf{Q}_{i, j, k}
\end{gathered}
$$

where

$$
\mathbf{M}=\frac{\mathbf{I}}{J \Delta t}+\mathbf{B}_{\xi}+\theta\left(\mathbf{B}_{\eta}+\mathbf{B}_{\zeta}\right)-\mathbf{S}_{\mathbf{Q}}
$$

and $\theta$ is a parameter that determines the order of accuracy of the time derivative and also can be used to control the stability of the scheme. A value of 2 is suggested in Ref. 51 and Ref. 52 and is used here as well. Note that the diagonal term $\mathbf{M}$ contains the diagonal contributions of $\delta_{\xi} \mathbf{E}_{\mathbf{Q}}, \delta_{\eta} \mathbf{F}_{\mathbf{Q}}, \delta_{\zeta} \mathbf{F}_{\mathbf{Q}}$ as well as $\mathbf{S}_{\mathbf{Q}}$ and is shared by both factors. This relaxation scheme could also be written as a conventional two factor scheme ${ }^{18}$ where the $\mathbf{B}_{\eta}$ and $\mathbf{B}_{\zeta}$ terms are removed from $\mathbf{M}$ and combined with their $\mathbf{A}$ and $\mathbf{C}$ contributions. However, this would increase factorization error without reducing cost or storage appreciably.

A three factor approximate factorization method that has lower storage requirements may be written

$$
\begin{gathered}
{\left[\mathbf{M}+\left(\mathbf{A}_{\xi}+\mathbf{B}_{\xi}+\mathbf{C}_{\xi}\right)\right] \Delta \mathbf{Q}_{i, j, k}^{*}=-\mathbf{R}^{n}} \\
{\left[\mathbf{M}+\left(\mathbf{A}_{\eta}+\mathbf{B}_{\eta}+\mathbf{C}_{\eta}\right)\right] \Delta \mathbf{Q}_{i, j, k}^{* *}=\mathbf{M} \Delta \mathbf{Q}_{i, j, k}^{*}} \\
{\left[\mathbf{M}+\mathbf{A}_{\zeta}+\mathbf{B}_{\zeta}+\mathbf{C}_{\zeta}\right] \Delta \mathbf{Q}_{i, j, k}=\mathbf{M} \Delta \mathbf{Q}_{i, j, k}^{* *}} \\
\mathbf{Q}_{i, j, k}^{n+1}=\mathbf{Q}_{i, j, k}^{n}+\Delta \mathbf{Q}_{i, j, k}
\end{gathered}
$$

where

$$
\mathbf{M}=\frac{\mathbf{I}}{J \Delta t}-\mathbf{S}_{\mathbf{Q}}
$$

Both factorized schemes above result in a tridiagonal system of block $N \times N$ matrices which must be inverted in either the two $(\eta, \zeta)$ or the three $(\xi, \eta, \zeta)$ directions. Note that Eq. (50) contains only the source term Jacobian.

The schemes described above are storage and operation intensive. A third scheme commonly referred to as a diagonalized approximate factorization (DAF) scheme $^{50}$ significantly reduces the storage and number of operations by diagonalizing the Jacobians and reducing the $N \times N$ block tridiagonals to a system of $N$ scalar tridiagonals. The DAF scheme may be written as

$$
\begin{gathered}
{\left[\mathbf{I}-J \Delta t \mathbf{S}_{\mathbf{Q}}\right] \Delta \mathbf{Q}_{i, j, k}^{*}=-J \Delta t \mathbf{R}^{n}} \\
\mathbf{T}_{\xi}\left[\mathbf{I}+J \Delta t \delta_{\xi}\left(\lambda_{\xi, c}-\lambda_{\xi, v}\right)\right] \mathbf{T}_{\xi}^{-1} \Delta \mathbf{Q}_{i, j, k}^{* *}=\Delta \mathbf{Q}_{i, j, k}^{*} \\
\mathbf{T}_{\eta}\left[\mathbf{I}+J \Delta t \delta_{\eta}\left(\lambda_{\eta, c}-\lambda_{\eta, v}\right)\right] \mathbf{T}_{\eta}^{-1} \Delta \mathbf{Q}_{i, j, k}^{* * *}=\Delta \mathbf{Q}_{i, j, k}^{* *} \\
\mathbf{T}_{\zeta}\left[\mathbf{I}+J \Delta t \delta_{\zeta}\left(\lambda_{\zeta, c}-\lambda_{\zeta, v}\right)\right] \mathbf{T}_{\zeta}^{-1} \Delta \mathbf{Q}_{i, j, k}=\Delta \mathbf{Q}_{i, j, k}^{* * *} \\
\mathbf{Q}_{i, j, k}^{n+1}=\mathbf{Q}_{i, j, k}^{n}+\Delta \mathbf{Q}_{i, j, k}
\end{gathered}
$$

where $\mathbf{T}_{\alpha}$ and $\mathbf{T}_{\alpha}^{-1}$ are matrices of the left and right eigenvectors, $\lambda_{\alpha, c}$ are the inviscid eigenvalues, and $\lambda_{\alpha, \nu}$ are a diagonalized form of the viscous eigenvalues.

\section{Space Marched Equations}

The Navier Stokes equation, Eq. (1), can be made parabolic in space while remaining hyperbolic in time by dropping all diffusive terms in the streamwise $(\xi)$ flux vector and by employing Vigneron's ${ }^{1}$ treatment of the streamwise pressure gradient terms. This results in an equation of the form

$$
\mathbf{Q}_{t}+\mathbf{E}_{\xi}+\left(\omega_{v}-1\right) \check{\mathbf{E}}_{\xi}^{\omega_{v}}+\left(\mathbf{F}-\mathbf{F}_{v}\right)_{\eta}+\left(\mathbf{G}-\mathbf{G}_{v}\right)_{\zeta}=\mathbf{S}
$$

where

$$
\check{\mathbf{E}}^{\omega_{v}}=\frac{|\nabla \xi|}{J}\left[\begin{array}{c}
0 \\
\vdots \\
0 \\
\hat{\xi}_{x} \bar{p} \\
\hat{\xi}_{y} \bar{p} \\
\hat{\xi}_{z} \bar{p} \\
0 \\
0 \\
0 \\
0 \\
0 \\
0
\end{array}\right]
$$


The $\left(\omega_{v}-1\right) \check{\mathbf{E}}_{\xi}^{\omega_{v}}$ term that appears in Eq. (52) is a correction to the streamwise flux difference, $\hat{\mathbf{E}}_{\xi}$, that parabolizes the streamwise pressure gradient. This correction term contains Vigneron's streamwise pressure-gradient coefficient, $\omega_{v}$, which is constrained such that the hyperbolic-parabolic nature of the governing equations is preserved with respect to the streamwise direction. The constraint equation that Vigneron obtained from an eigenvalue analysis is

$$
\begin{gathered}
\omega_{v}=\min \left[1, \frac{\sigma \gamma M_{\xi}^{2}}{1+(\gamma-1) M_{\xi}^{2}}\right] \\
M_{\xi}^{2}=\frac{U^{2}}{\left(\xi_{x}^{2}+\xi_{y}^{2}+\xi_{z}^{2}\right) \gamma \bar{p} / \bar{\rho}}
\end{gathered}
$$

where $\gamma$ is the frozen ratio of specific heats and $\sigma$ is a safety factor (usually $=0.95$ ) included to account for nonlinearities that are not considered in the eigenvalue analysis. $^{1}$

Eq. (52) is written in a correction form in a manner similar to Morrison and Korte. ${ }^{19}$ Morrison and Korte found that the implementation of Vigneron's streamwise pressure gradient approximation in a cell centered finite volume context required special treatment to avoid the introduction of artificial pressure gradient terms which in turn cause errors that are strongly grid dependent. As in Morrison and Korte, ${ }^{19} \omega_{v}$ is evaluated at the cell center by averaging the cell face metrics and cell face primitive variables obtained from either a first or second order fully upwind reconstruction.

\section{Space Marching Solution Procedures}

Following Newsome et al., ${ }^{18}$ Eq. (52) is solved by constructing the fluxes and source terms for a constant $\xi$ plane (three-dimensional) or line (two-dimensional and axisymmetric) of cells and then applying the forward sweep step of the planar Gauss-Seidel relaxation scheme presented in Eq. (45). Eq. (45) is modified slightly for the space marching procedure to become

$$
\begin{gathered}
{\left[\frac{\mathbf{I}}{J \Delta t}+\mathbf{B}_{\xi}^{\omega_{c}}+\mathbf{B}_{\eta}+\mathbf{B}_{\zeta}-\mathbf{S}_{\mathbf{Q}}\right] \Delta \mathbf{Q}_{i, j, k}+} \\
\mathbf{A}_{\eta} \Delta \mathbf{Q}_{i, j-1, k}+\mathbf{C}_{\eta} \Delta \mathbf{Q}_{i, j+1, k}+ \\
\mathbf{A}_{\zeta} \Delta \mathbf{Q}_{i, j, k-1}+\mathbf{C}_{\zeta} \Delta \mathbf{Q}_{i, j, k+1}= \\
-\mathbf{R}^{n}\left(\mathbf{Q}_{i-2, j, k}^{n+1}, \mathbf{Q}_{i-1, j, k}^{n+1}, \mathbf{Q}_{i, j, k}^{n}\right)
\end{gathered}
$$

where $\mathbf{B}_{\xi}^{\omega_{c}}$ is the Jacobian of the parabolized streamwise flux, $\mathbf{E}_{\xi}+\left(\omega_{v}-1\right) \check{\mathbf{E}}_{\xi}^{\omega_{v}}$.

Extending the approach of Newsome et al. to include source terms, the cross-stream plane is factored using a two factor scheme

$$
\begin{gathered}
{\left[\mathbf{M}+\left(\mathbf{A}_{\eta}+\mathbf{B}_{\eta}+\mathbf{C}_{\eta}\right)\right] \Delta \mathbf{Q}_{i, j, k}^{*}=-\mathbf{R}^{n}} \\
{\left[\mathbf{M}+\left(\mathbf{A}_{\zeta}+\mathbf{B}_{\zeta}+\mathbf{C}_{\zeta}\right)\right] \Delta \mathbf{Q}_{i, j, k}=\mathbf{M} \Delta \mathbf{Q}_{i, j, k}^{*}} \\
\mathbf{Q}_{i, j, k}^{n+1}=\mathbf{Q}_{i, j, k}^{n}+\Delta \mathbf{Q}_{i, j, k}
\end{gathered}
$$

and

$$
\mathbf{M}=\frac{\mathbf{I}}{J \Delta t}+\mathbf{B}_{\xi}^{\omega_{c}}-\mathbf{S}_{\mathbf{Q}}
$$

However, an approach that has less factorization error is to factor Eq. (56) in the cross stream plane using DDADI

$$
\begin{gathered}
{\left[\mathbf{M}+\theta\left(\mathbf{A}_{\eta}+\mathbf{C}_{\eta}\right)\right] \Delta \mathbf{Q}_{i, j, k}^{*}=-\mathbf{R}^{n}} \\
{\left[\mathbf{M}+\theta\left(\mathbf{A}_{\zeta}+\mathbf{C}_{\zeta}\right)\right] \Delta \mathbf{Q}_{i, j, k}=\mathbf{M} \Delta \mathbf{Q}_{i, j, k}^{*}} \\
\mathbf{Q}_{i, j, k}^{n+1}=\mathbf{Q}_{i, j, k}^{n}+\Delta \mathbf{Q}_{i, j, k}
\end{gathered}
$$

and

$$
\mathbf{M}=\frac{\mathbf{I}}{J \Delta t}+\mathbf{B}_{\xi}^{\omega_{c}}+\theta\left(\mathbf{B}_{\eta}+\mathbf{B}_{\zeta}\right)-\mathbf{S}_{\mathbf{Q}}
$$

The DDADI factorization is an expensive algorithm, especially when the number of equations is large. Therefore, a modified planar Gauss-Seidel relaxation which uses the diagonalized approximate factorization scheme is proposed that has lower storage and operation requirements for use on moderate aspect ratio grids. This scheme will converge poorly when the streamwise grid aspect ratio becomes large; however, by combining the DAF factorization with wall matching functions, the wall normal grid spacing (and thus aspect ratio) can be relaxed and acceptable convergence behavior recovered. A planar relaxation based on DAF may be written as

$$
\begin{gathered}
{\left[\mathbf{I}+J \Delta t\left(\mathbf{B}_{\xi}^{\omega_{c}}-\mathbf{S}_{\mathbf{Q}}\right)\right] \Delta \mathbf{Q}_{i, j, k}^{*}=-J \Delta t \mathbf{R}^{n}} \\
\mathbf{T}_{\eta}\left[\mathbf{I}+J \Delta t \delta_{\eta}\left(\lambda_{\eta, c}-\lambda_{\eta, v}\right)\right] \mathbf{T}_{\eta}^{-1} \Delta \mathbf{Q}_{i, j, k}^{* *}=\Delta \mathbf{Q}_{i, j, k}^{*} \\
\mathbf{T}_{\zeta}\left[\mathbf{I}+J \Delta t \delta_{\zeta}\left(\lambda_{\zeta, c}-\lambda_{\zeta, v}\right)\right] \mathbf{T}_{\zeta}^{-1} \Delta \mathbf{Q}_{i, j, k}=\Delta \mathbf{Q}_{i, j, k}^{* *} \\
\mathbf{Q}_{i, j, k}^{n+1}=\mathbf{Q}_{i, j, k}^{n}+\Delta \mathbf{Q}_{i, j, k}
\end{gathered}
$$

Either of the relaxation procedures given above is then used to solve each $\xi$ plane of cells beginning at the inflow plane and sweeping in positive $\xi$ through the computational domain. Each $\xi$ plane of cells is iterated in pseudo-time until convergence is reached (a four order reduction in the sum of the $L_{2}$ norm of the continuity, momentum, and energy residuals is generally sufficient). The solution is then projected into the next plane of cells as an initial condition. This procedure is repeated for each plane of cells until the outflow plane is reached.

\section{Convergence Acceleration}

VULCAN has three techniques for accelerating the convergence of Eqs. (1) and (52). The first technique is local time stepping, the second technique is coarseto-fine grid sequencing, and the third technique is the Full Approximate Storage (FAS) multi-grid scheme of Brandt. 53 
The FAS grid transfer operators for the solution, residual, and coarse grid corrections are those introduced by Jameson. ${ }^{54}$ The multi-grid strategy can be either a $\mathrm{V}(1,0)$ or $\mathrm{W}(1,0)^{48}$ type cycle. The corrections after each prolongation are smoothed using a scalar coefficient implicit residual smoothing to remove high frequency errors introduced by the prolongation operator. To provide a sufficiently smooth initial condition for the fine grid, the Full Multi-grid (FMG) method can be invoked. The use of FMG is similar to coarse-to-fine grid sequencing with the exception that multi-grid cycles are performed on each level of the coarse-to-fine grid sequence.

The multi-grid scheme also has several features that are not commonly employed. The governing equations are all solved in a fully coupled fashion on all grid levels. Additionally, it was found useful for higher Mach number flows to construct the viscous fluxes as well as the source terms on all coarse grids. The turbulence model equations are solved on all grid levels; however, some special treatment for wall boundary conditions are employed. The wall boundary condition on the dissipation equation is either restricted from the fine grid or computed on the coarse grid by evaluating the wall matching function on the coarse grid.

Implementation of the FAS and FMG methods is identical for the hyperbolic-elliptic and the hyperbolicparabolic forms of the equations with one exception. When solving the hyperbolic-elliptic form of the equation, the grid is coarsened in all directions uniformly. However, when solving the hyperbolic-parabolic form of the equations, the grid is coarsened in the cross flow plane only. For two-dimensional and axisymmetric problems this results in grid coarsening in the $\eta$ direction only; while for three-dimensional problems, this results in coarsening in the $\eta$ and $\zeta$ directions only.

\section{Results}

Four example problems are presented to demonstrate the capabilities of the multi-grid, space marching, relaxation algorithm. The first test case is inviscid, calorically perfect, Mach 2 flow over a twodimensional bump in a channel; the second is calorically perfect, turbulent, viscous, Mach 6 flow over a flat plate; the third is calorically perfect, laminar, Mach 3 flow over a three dimensional compression corner; and the fourth is a two-dimensional supersonic turbulent diffusion flame.

Figure 1 shows the two-dimensional computational grid for a Mach 2 simulation of inviscid flow over a bump in a channel. The grid consists of 64 cells in the streamwise $(\xi)$ direction and 32 cells in the cross flow $(\eta)$ direction. The grid aspect ratio can be seen to be nearly one for all cells and the grid is orthogonal to the lower boundary. Figure 2 presents density contours from a space marched solution obtained using line Gauss-Seidel relaxation. The streamwise fluxes were constructed using second order, fully upwind extrapolation and the cross-stream fluxes were constructed using MUSCL with $\kappa=1 / 3$ and the smooth limiter of Venkatakrishnan. ${ }^{45}$ A grid sub-stepping feature available in VULCAN was employed to control oscillations near the shock. Space marching results in a lack of downstream information during the streamwise flux construction making it impossible to employ flux limiters in the "classical" sense. However, VULCAN can be made to "sub-step" on the grid. Substepping is a procedure that linearly sub-divides the input streamwise cells, thereby reducing the gradients in the streamwise direction and increasing accuracy while also reducing oscillations. Figure 3 shows the typical convergence behavior of several computational planes from the solution. Each plane was initialized with the solution from the previous plane and the CFL was ramped from an initial value of 100 to a final value of 1000 over 5 iterations. All planes converged 4 orders in the $L_{2}$ norm in 15 iterations or less. Multi-grid was not found to appreciably improve the already excellent convergence rate of this problem. The problem was also run using the DAF based Gauss-Seidel scheme using a CFL ramped from 1 to 10 over 5 iterations. The DAF scheme required 20 iterations on average to reduce the $L_{2}$ four orders of magnitude. However, the DAF scheme had approximately the same cost per plane due to its lower cost per iteration.

Figure 4 presents the Mach contours from a supersonic, calorically perfect $(\gamma=1.4)$ calculation of turbulent flow over a 0.5 meter long flat plate. The unit free stream Reynolds number is $2.64 \times 10^{7}$ and the wall temperature ratio $T_{w} / T_{\infty}=0.65$. The baseline $\tilde{k}-\tilde{\omega}$ turbulence model was solved to the wall on a computational grid of 200 cells in the $\xi$ direction and 153 cells in the $\eta$ direction. The streamwise fluxes were constructed using second order, fully upwind extrapolation and the cross-stream fluxes were constructed using MUSCL with $\kappa=1 / 3$ and the smooth limiter of Venkatakrishnan. ${ }^{45}$ The $y^{+}$of the first cell center off the wall was less than 0.5 for the entire plate. Figure 5 provides the computed outflow velocity and eddy viscosity profile demonstrating that the boundary layer is well resolved. Figure 6 presents several typical "posttransition" convergence histories at a location where the grid aspect ratio was $\approx 300$. The line Gauss-Seidel relaxation space marching scheme was used with a W $(1,0)$ cycle FMG with 3 grid levels. The $L_{2}$ norm of the residual is reduced 12 orders of magnitude on each streamwise step in 32 multi-grid cycles. Although not shown, it is important to note that the residual of the $\tilde{k}$ and $\tilde{\omega}$ equations both converged at the same rate and to the same level as shown here. The problem was run without multi-grid and was found to require more than 130 cycles to converge to the same level as shown in figure 6 .

The third case is the supersonic laminar three- 
dimensional flow over the symmetric corner compression flow of Korkegi and West. ${ }^{55}$ The unit free stream Reynolds number for this problem is $3.07 \times 10^{6}$ and the wall temperature ratio is $T_{w} / T_{\infty}=1.00$. The computational grid, shown in Figure 7 , has 64 cells in the $\xi$ direction and 128 cells in both the $\eta$ and $\zeta$ directions for a total $1,048,576$ cells. The grid was constructed such that the streamwise grid aspect ratio was 300 at the inflow plane and grew to 10,000 at the outflow plane so that the sensitivity of the algorithm to grid aspect ratio could be examined. The streamwise fluxes were constructed using second order, fully upwind extrapolation and the cross-stream fluxes were constructed using MUSCL with $\kappa=1 / 3$ and the smooth limiter of Venkatakrishnan. ${ }^{45}$ A planar GaussSeidel relaxation which used a DDADI cross-stream factorization was used to drive a V $(1,0)$ cycle FMG with 3 grid levels. Figure 8 presents density contours of the corner interaction region that was computed illustrating the self similar nature of the flow. Figure 9 presents the convergence history where the grid aspect ratio was on the order of 7000 . The CFL number was ramped from 100 to 1000 on each plane over 25 cycles. The method did exhibit some dependence on grid aspect ratio. Initially, where the grid aspect ratio was 300 , the solution required on the order of $30-40$ multi-grid cycles per plane. However, the number of cycles per plane gradually climbed until it stabilized at 50 cycles per plane. As shown in figure 9 the $L_{2}$ norm of the residual was reduced by four orders in approximately 50 multi-grid cycles. When the grid aspect ratio exceeded 400 , the fine grid scheme without multi-grid required more than five times as many cycles to achieve the same level of convergence.

The final example is a two-dimensional turbulent diffusion flame which was calculated by solving the flow over an infinitely thin splitter plate that separates pure hydrogen flowing at $3800 \mathrm{~m} / \mathrm{sec}$ (with a pressure of 1 atmosphere and a temperature of 1000 $\mathrm{K}$ ), from a mixture of $\mathrm{O}_{2}$ and $\mathrm{N}_{2}$ flowing at 1200 $\mathrm{m} / \mathrm{sec}$ (with a pressure of 1 atmosphere and a temperature of $1000 \mathrm{~K}$ ) and then solving the wake and shear layer that form when the fuel and oxidizer mix. The flow was space marched from the plate leading edge to the plate trailing edge solving the Menter's baseline two-equation turbulence model equations to the adiabatic wall. The flow was then space marched downstream from the plate trailing edge where it develops as a wake and then as a shear layer. Figure 10 shows the three block computational grid that was used. Blocks one and two consisted of 64 cells in the $\xi$ direction and $\eta$ direction and block three contained 96 cells in the $\xi$ direction and 128 cells in the $\eta$ direction. Blocks one and two were run non-reacting and block three was run reacting with a 7 species, 7 reaction model. ${ }^{40}$ Figures 11 and 12 present Mach and water contours showing the boundary and shear layers as well as the flame location. The fuel and oxidizer can be seen to have mixed in the shear layer and the flame auto-ignited a short distance downstream of the plate trailing edge. The computation was made using the DAF line Gauss-Seidel scheme with a V $(1,0)$ FMG cycle with 3 grid levels. Blocks one and two required between 70 and 100 multi-grid cycles per streamwise step; block three required on the order of 100 multigrid cycles per streamwise step to reduce the $L_{2}$ norm of the residual four orders of magnitude. Typical convergence histories are shown for each of the blocks in figures 13,14 , and 15 .

\section{Concluding Remarks}

The multi-grid, flux-difference split, finite-volume code, VULCAN, that solves the elliptic and parabolized forms of the equations governing threedimensional, turbulent, calorically perfect and nonequilibrium chemically reacting flows was described. Space marching algorithms utilizing multi-grid to accelerate convergence were demonstrated. In addition, a Gauss-Seidel planar relaxation scheme based on diagonalized approximate factorization was introduced and demonstrated. This algorithm was shown to reduce cost and storage relative to the diagonally dominant alternating direction implicit scheme (DDADI). The convergence rate of the full-approximate storage (FAS) multi-grid scheme applied to the parabolized Navier-Stokes equations for calorically perfect and non-equilibrium chemically reacting flows was demonstrated and found to perform well on high aspect ratio grids.

\section{Acknowledgements}

The multi-grid relaxation scheme development was jointly supported by the Air Force Research Laboratory at Wright-Patterson Air Force Base and NASA Langley Research Center. Special thanks go to Dr. Jack R. Edwards of North Carolina State University Dr. R. Charles Swanson of NASA Langley Research Center, and Dr. Richard L. Gaffney of Analytical Services and Materials, Inc. for their contributions to this work.

\section{References}

\footnotetext{
${ }^{1}$ Vigneron, Y.C., Rakich, J.V. and Tannehill, J.C., "Calculation of Supersonic Viscous Flow Over Delta Wings With Sharp Subsonic Leading Edges," AIAA 78-1137, 1978.

${ }^{2}$ Davis, R.T., Barnett, M. and Rackich, J.V., "The Calculation of Supersonic Viscous Flows Using the Parabolized Navier-Stokes Equations," Computer and Fluids, Vol. 14, No. 3, pp. 197-224, March 1986.

${ }^{3}$ Gielda, T.P. and McRae, D.S., "An Accurate, Stable, Explicit Parabolized Navier-Stokes Solver For High Speed Flows," AIAA 86-1116, 1986

${ }^{4}$ Lawrence, S.C., Tannehill, J.C. and Chausse, D.S., "An Upwind Algorithm for the Parabolized Navier-Stokes Equations," AIAA 86-1117, 1986.
} 
${ }^{5}$ Korte, J.J. and McRae, D.S., "Explicit Upwind Algorithm for the Parabolized Navier-Stokes Equations," AIAA 88-0716, 1988 .

${ }^{6}$ Korte, J.J. and McRae, D.S., "Numerical Simulation of Flow Over a Hypersonic Aircraft Using an Explicit Upwind PNS Solver," AIAA 89-1829, 1989.

${ }^{7}$ Gerbsch, R.A. and Agarwal, R.K., "Solutions of the Parabolized Navier-Stokes Equations Using Osher's Upwind Scheme," AIAA 90-0932, 1990.

${ }^{8}$ Gielda, T.P., Hunter, L.G. and Chawner, J.R., "Efficient Parabolized Navier-Stokes Solutions of Three Dimensional, Chemically Reacting Flows," AIAA 88-0096, 1988.

${ }^{9}$ Kamath, H., "Parabolized Navier-Stokes Algorithm for Chemically Reacting Flows," AIAA 89-0386, 1989.

${ }^{10}$ Gerbsch, R.A. and Agarwal, R.K., "Solution of the Parabolized Navier-Stokes Equations For Three-Dimensional Real-Gas Flows Using Osher's Upwind Scheme," AIAA 91-0248, 1991.

${ }^{11}$ Hugues, E. and Verant, J.L., "Nonequilibrium Parabolized Navier-Stokes Code with an Implicit Finite Volume Method," AIAA 91-0470, 1991.

${ }^{12}$ Tannehill, J.C., Ievalts, J.O. and Lawrence, S.L., "An Upwind Parabolized Navier-Stokes Code for Real Gas Flows," AIAA 88-0713, 1988.

13 Tannehill, J.C., Ievalts, J.O., Buelow, P.E., Prabhu, D.K. and Lawrence, S.L., "An Upwind Parabolized Navier-Stokes Code for Chemically Reacting Flows," Journal of Thermophysics and Heat Transfer, Vol. 4, No. 2, pp. 149-156, April 1990 .

${ }^{14}$ Sinha, N., Dash, S.M. and Lee, R.A., "3-D PNS Analysis of Scramjet Combustion/Nozzle and Exhaust Plume Flows," AIAA 90-0094, 1990.

${ }^{15}$ Tannehill, J.C., Ievalts, J.O. and Lawrence, S.L., "A ThreeDimensional Upwind PNS Code for Chemically Reacting Scramjet Flowfields," AIAA 92-2898, 1992.

${ }^{16}$ Korte, J.J., "Aerodynamic Design of Axisymmetric Hypersonic Wind Tunnel Nozzles Using Least-Squares/Parabolized Navier-Stokes Procedure," AIAA 92-0332, 1992.

${ }^{17}$ Evans, J.R., Wardlaw, A.B. Jr. and Davis, S.F., "NSWC/DREV 3D Explicit Parabolized Navier-Stokes Code," Defense Research Establishment of Canada, DREV report 4669/92, January, 1992.

${ }^{18}$ Newsome, R.W., Walters, R.W. and Thomas, J.L., "An Efficient Iteration Strategy For Upwind/Relaxation Solutions to the Thin-Layer Navier-Stokes Equations," AIAA 87-1113, 1987.

${ }^{19}$ Morrison, J.H. and Korte, J.J., "Implementation of Vigneron's Streamwise Pressure Gradient Approximation in the PNS Equations," AIAA 92-0189, 1992.

${ }^{20}$ van Leer, B., "Towards the Ultimate Conservative Difference Scheme V. A Second Order Sequel to Gudunov's Method," Journal of Computational Physics, Vol. 32, pp. 101-136, 1979.

${ }^{21}$ Roe, P.L., "Discrete Models for the Numerical Analysis of Time-Dependent Multidimensional Gas Dynamics," Journal of Computational Physics, Vol. 63, pp. 458-476, 1986.

${ }^{22}$ Edwards, J.R., "A Low-Diffusion Flux-Splitting Scheme for Navier-Stokes Calculations," Computers and Fluids, Vol. 26. No. 6, pp. 635-659, June 1997

${ }^{23}$ White, J.A., Korte, J.J., and Gaffney, R.L. Jr., "FluxDifference Split Parabolized Navier-Stokes Algorithm for Nonequilibrium Chemically Reacting Flows," AIAA 93-0534, 1993.

${ }^{24}$ Menter, F.R., "Improved Two-Equation k- $\omega$ Turbulence Model for Aerodynamic Flows," NASA TM-103975, 1992.

${ }^{25}$ Abid, R., Morrison, J.H., Gatski, T.B., and Speziale, C.G., "Prediction of Complex Aerodynamic Flows with Explicit Reynolds Stress Models," AIAA 96-0565, 1996.

${ }^{26}$ Gatski, T.B. and Speziale, C.G., "On Explicit Algebraic Stress Models for Complex Turbulent Flows," NASA-CR189725 .
27 Abid, R., Rumsey, C.L., and Gatski, T.B., "Prediction of Nonequilibrium Turbulent Flows with Explicit Algebraic Turbulence Models," AIA A Journal, Vol. 33, No. 11, Nov. 1995.

${ }^{28}$ Wilcox, D.C., Turbulence Modeling for $C F D$, 2nd Edition, DCW Industries, Inc., 1998.

${ }^{29}$ Sarkar, S., Erlebacher, G., Hussaini, M.Y. and Kreiss, H.O., "The Analysis and Modeling of Dilatational Terms in Compressible Turbulence," ICASE Report 89-79, 1989.

${ }^{30}$ Zeman, O., "Dilatational Dissipation: The Concept and Application in Modeling Compressible Mixing Layers," Physics of Fluids, Vol. 2, No. 2, Feb. 1990.

${ }^{31}$ Abid, R., "Evaluation of Two-Equation Turbulence Models for Predicting Transitional Flows," International Journal Engineering Science, Vol. 31, No. 6, pp. 831-840, 1993.

${ }^{32}$ Wilcox, D.C., "Wall Matching, A Rational Alternative To Wall Functions," AIAA 89-0611, 1989.

${ }^{33}$ McBride, B.J., Heimel, S., Ehlers, J.G. and Gordon, S., "Thermodynamic Properties to $6000 \mathrm{~K}$ for 210 Substances Involving the First 18 Elements," NASA SP-3001, 1963.

${ }^{34}$ McBride, B.J., Gordon, S. and Reno, M.A., "Thermodynamic Data for Fifty Reference Elements," NASA TP-3287, 1993.

${ }^{35}$ Wilke, C.R., "A Viscosity Equation for Gas Mixtures," Chemical Physics, Vol. 18, No. 4, pp. 517-519, April 1950.

${ }^{36}$ Berman, H.A., Anderson, J.D. Jr., and Drummond, J.P., "Supersonic Flow Over a Rearward Facing Step With Transverse Nonreacting Hydrogen Injection," AIAA Journal, Vol. 21, No. 12, pp. 1707-1713, Dec. 1983.

${ }^{37}$ Drummond, J.P. and Hussaini, M.Y., "Numerical Simulation of a Supersonic Reacting Mixing Layer," AIAA 87-1235, 1987 .

${ }^{38}$ Drummond, J.P., Rogers, R.C. and Hussaini, M.Y., "A Numerical Model for Supersonic Reacting Mixing Layers," Computer Methods in Applied Mathematics and Engineering, Vol. 64, pp. 39-60, 1987.

${ }^{39}$ Williams, F.A., Combustion Theory, Second Ed., The Benjamin/Cummings Publishing Company, Inc., Menlo Park, California, 1985 .

${ }^{40}$ Carpenter, M.H., "A Generalized Chemistry Version of SPARK," NASA-CR-4196, 1988

${ }^{41}$ Gaffney, R.L. Jr., White, J.A., Girimaji, S.S. and Drummond, J.P., "Modeling Turbulent/Chemistry Interactions Using Assumed PDF Methods," AIAA 92-3638, 1992.

${ }^{42}$ Girimaji, S.S. "A Simple Recipe for Modeling Reaction Rates in Flows with Turbulent Combustion," AIAA 91-1792, 1992.

${ }^{43}$ Gaffney, R.L. Jr., White, J.A., Girimaji, S.S. and Drummond, J.P., "Modeling Temperature and Species Fluctuations In Turbulent, Reacting Flow," Computing Systems in Engineering, Vol. 5, No. 2, pp. 117-133, 1994.

${ }^{44}$ van Albada, G.D., van Leer, B. and Roberts, W.W., Jr., "A Comparative Study of Computational Methods in Cosmic Gas Dynamics," Astronomy and Astrophysics, Vol. 108, pp. 76-84, 1982.

${ }^{45}$ Venkatakrishnan, V., "On the Accuracy of Limiters and Convergence to Steady State Solutions," AIAA 93-0880, 1993.

${ }^{46}$ Chakravarthy, S.R., Szema, K-Y., Goldberg, U.C., and Gorski, J.J., "Application of a New Class of High Accuracy TVD Schemes to the Navier-Stokes Equations," AIAA 85-0165, 1985.

47 Jameson, A., Schmidt, W.. and Turkel, E., "Numerical Solutions of the Euler Equations by Finite Volume Methods with Runge-Kutta Time Stepping Schemes," AIAA 81-1259, 1981.

${ }^{48}$ Swanson, R. C. and Turkel, E., "Multistage Scheme with Multigrid for Euler and Navier-Stokes Equations: Components and Analysis," NASA Technical Paper 3631, 1997.

${ }^{49}$ Lombard, C.K., Bardina, J., and Venkatapathy, E., "Multidimensional Formulation of CSCM-An Upwind Flux Difference Eigenvector Split Method for the Compressible Navier-Stokes Equations," AIAA 83-1895, 1983. 
${ }^{50}$ Pulliam, T.H. and Chaussee, D.S., "A Diagonal Form of an Implicit Approximate Factorization Algorithm," Journal of Computational Physics, Vol. 39, p. 347, 1981.

${ }^{51}$ MacCormack, R.J. and Pulliam, T., "Assessment of a New Numerical Procedure for Fluid Dynamics," AIAA 998-2821, 1998.

${ }^{52}$ Klopfer, G.H., Hung, C.M., Van der Wijngaart, R.F., and Onufer, J.T., "A Diagonalized Diagonal Dominant Alternating Direction Implicit (D3ADI) Scheme And Subiteration Correction," AIAA 98-2824, 1998.

${ }^{53}$ Brandt. A., Multi-Level Adaptive Solutions to BoundaryValue Problems, Vol. 31, No. 138, pp. 333-390, April 1977.

54 Jameson, A. and Baker, T.J., "Multigrid Solution of the Euler Equations for Aircraft Configurations," AIAA 84-0093, 1984.

${ }^{55}$ West, J.E. and Korkegi, R.H., "Supersonic Interaction in the Corner of Intersecting Wedges at High Reynolds Numbers," AIAA Journal, Vol. 10, No. 5, May 1972.

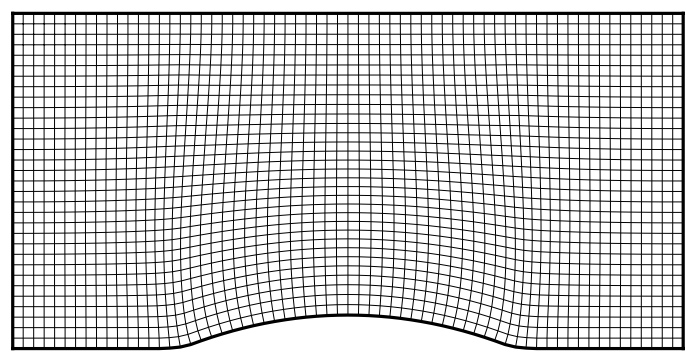

Fig. 1 Computational grid for a bump in a channel.

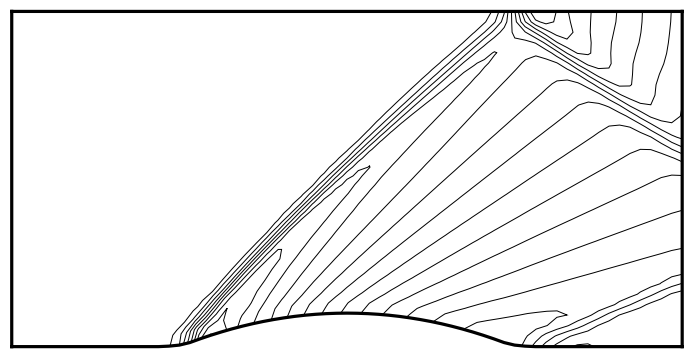

Fig. 2 Computed density contours for Mach 2

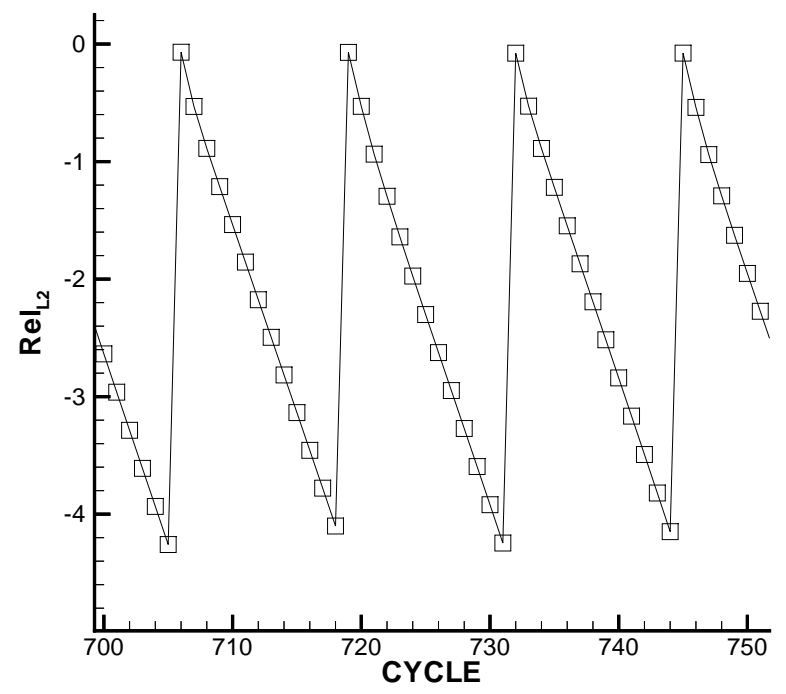

Fig. 3 Typical convergence for space marched bump.

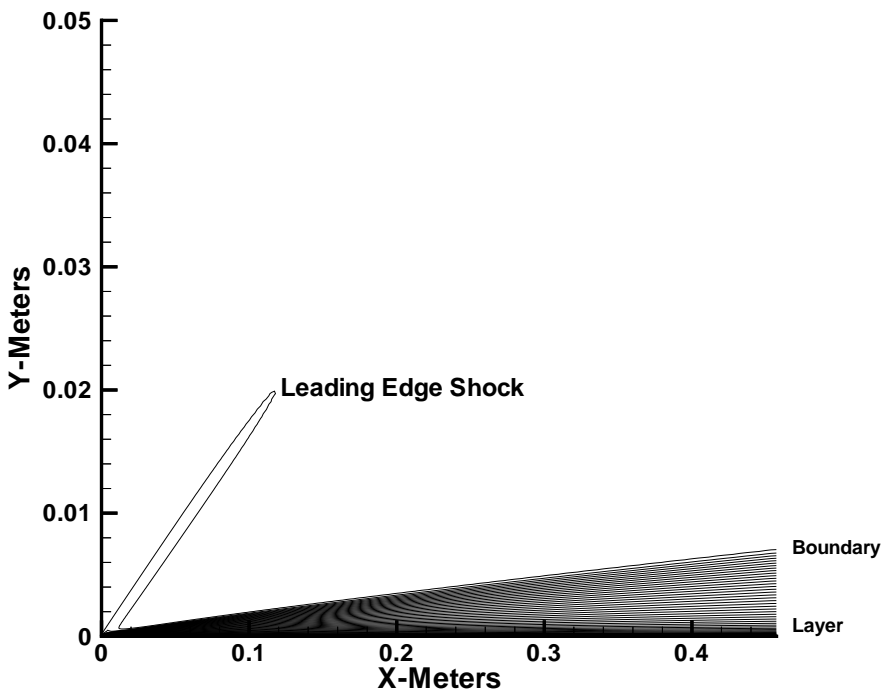

Fig. 4 Mach contours for Mach 6 turbulent flat plate flow. 


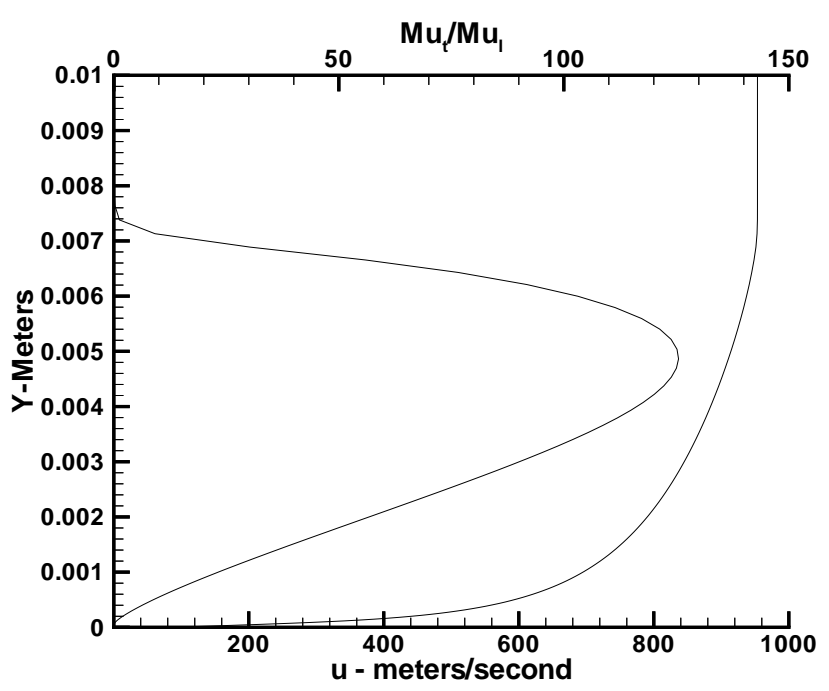

Fig. 5 Outflow profile for Mach 6 turbulent flatplate flow.

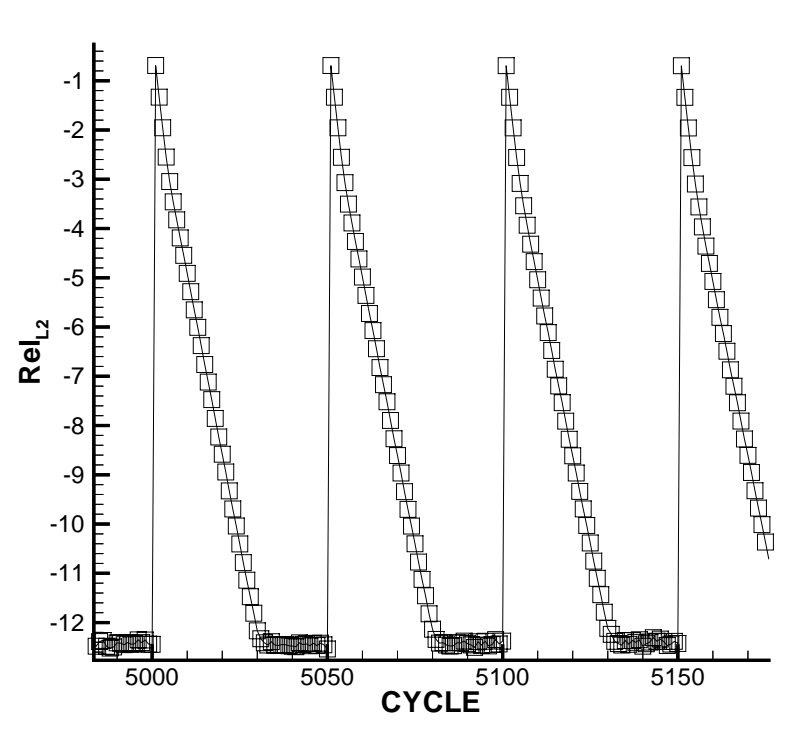

Fig. 6 Convergence history for Mach 6 turbulent flatplate.

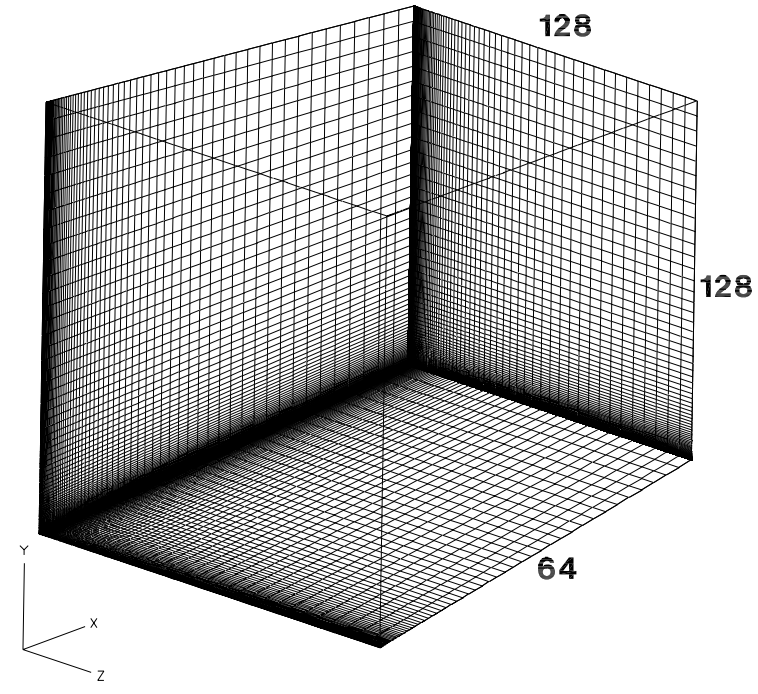

Fig. 7 Computational grid for supersonic symmetric corner flow.

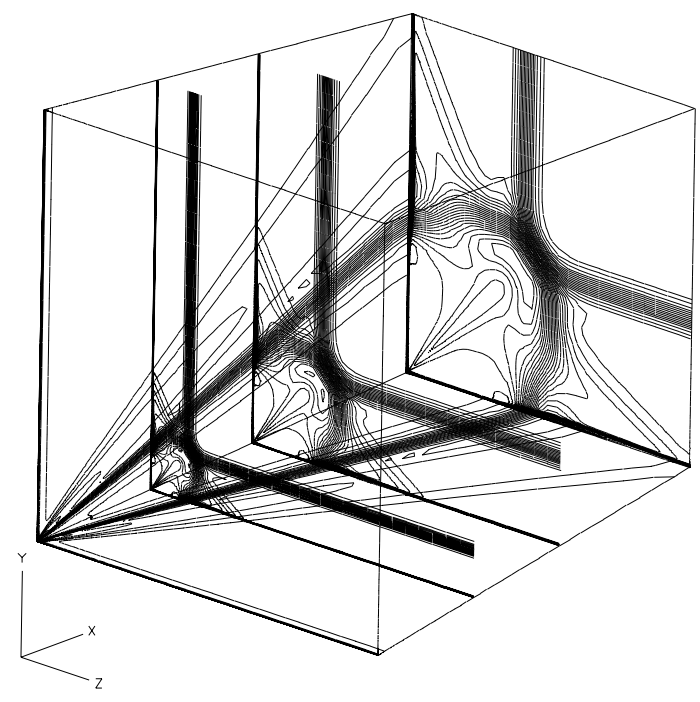

Fig. 8 Density contours for supersonic symmetric corner flow. 


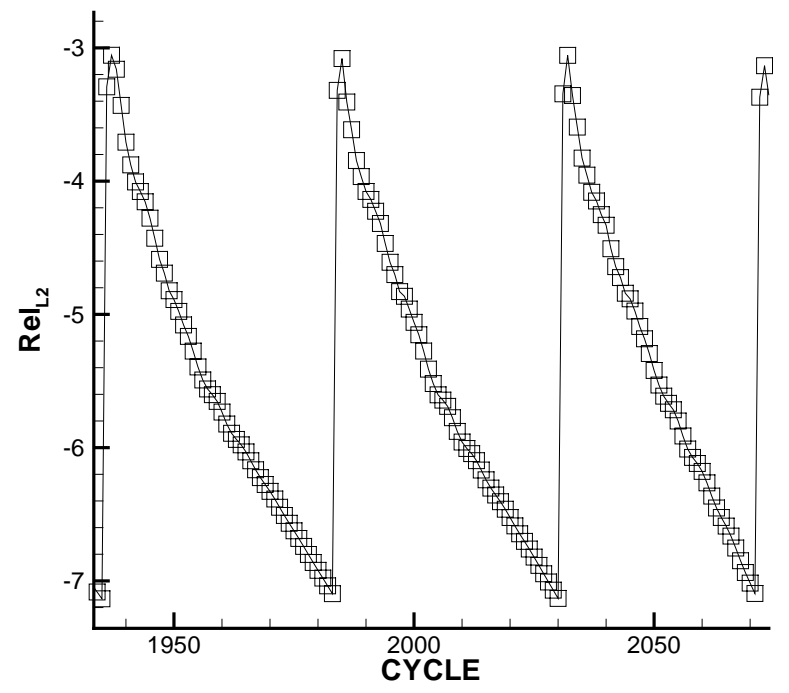

Fig. 9 Typical convergence for supersonic symmetric corner flow.

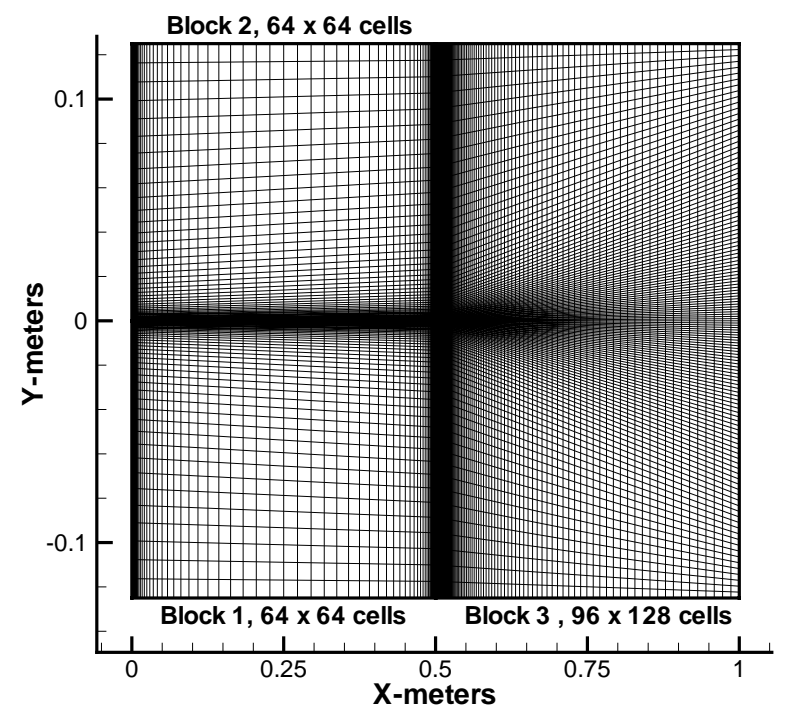

Fig. 10 Computational grid for turbulent diffusion flame.

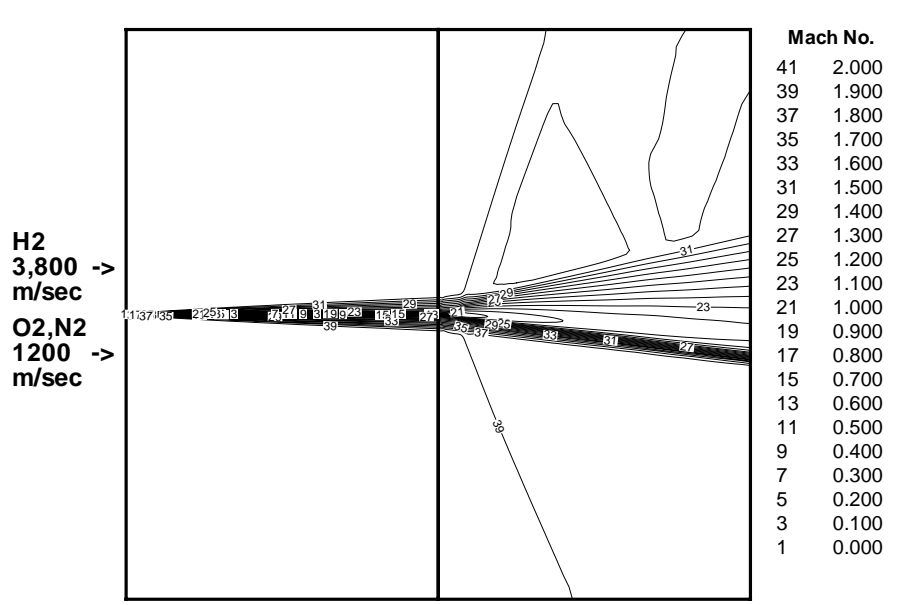

Fig. 11 Mach contours for turbulent diffusion flame.

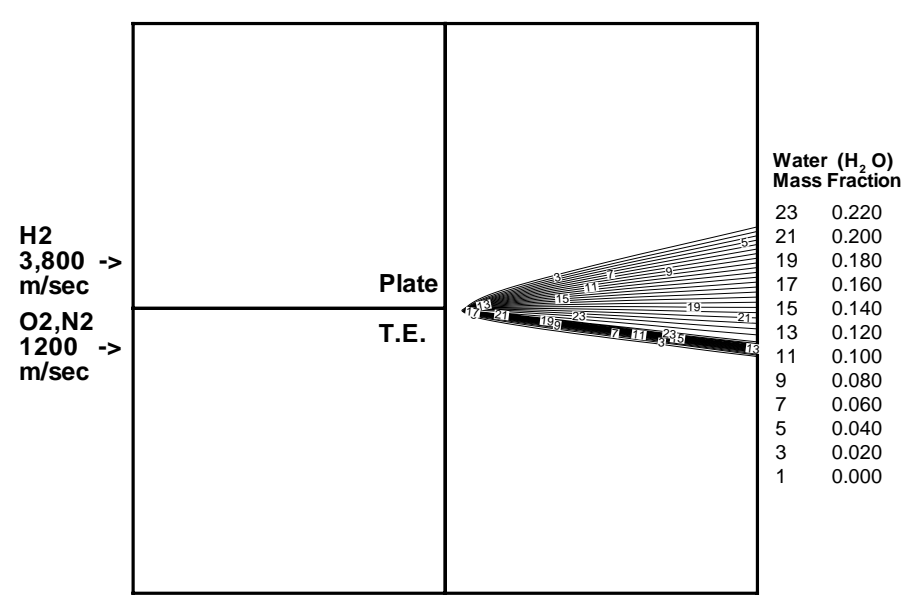

Fig. 12 Water contours for turbulent diffusion flame. 


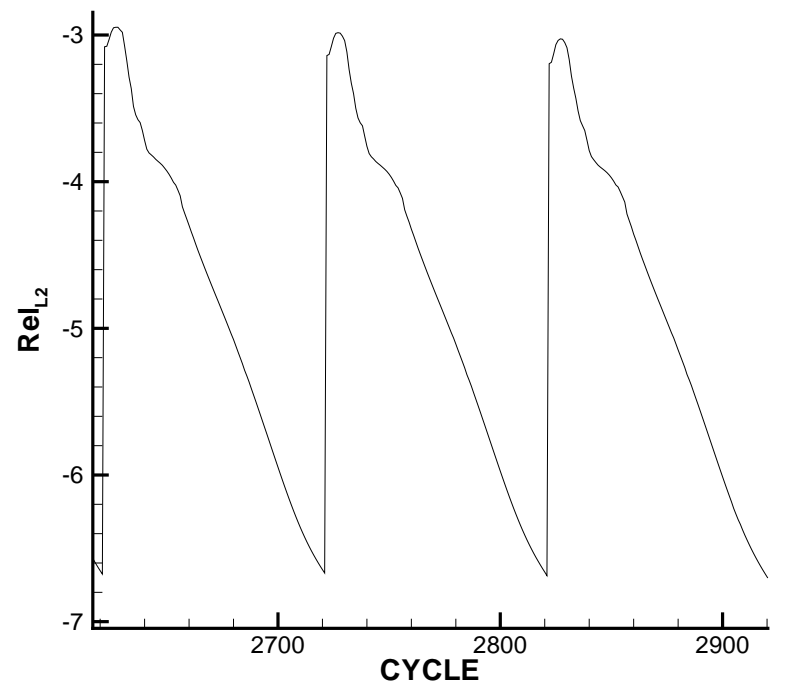

Fig. 13 Convergence for Block 1 of turbulent diffusion flame.

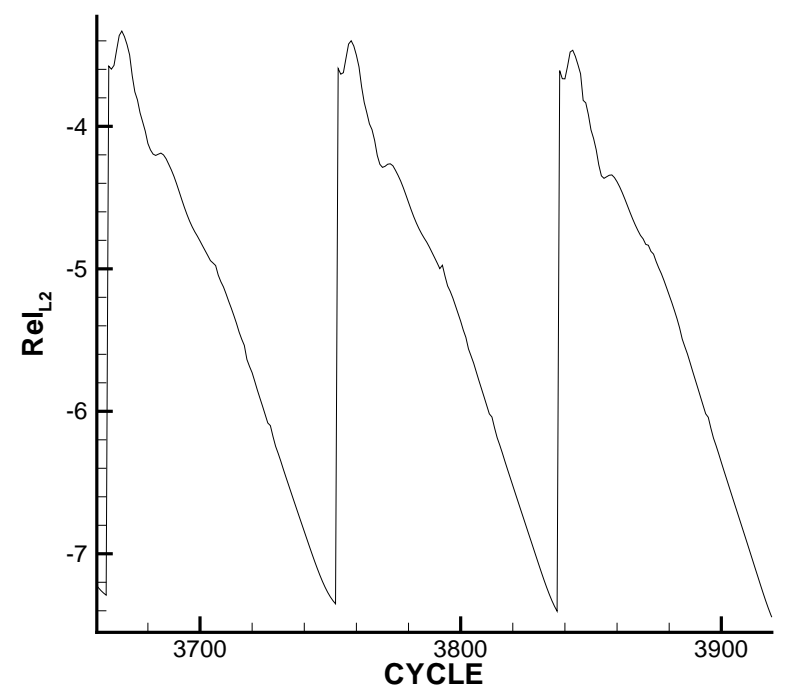

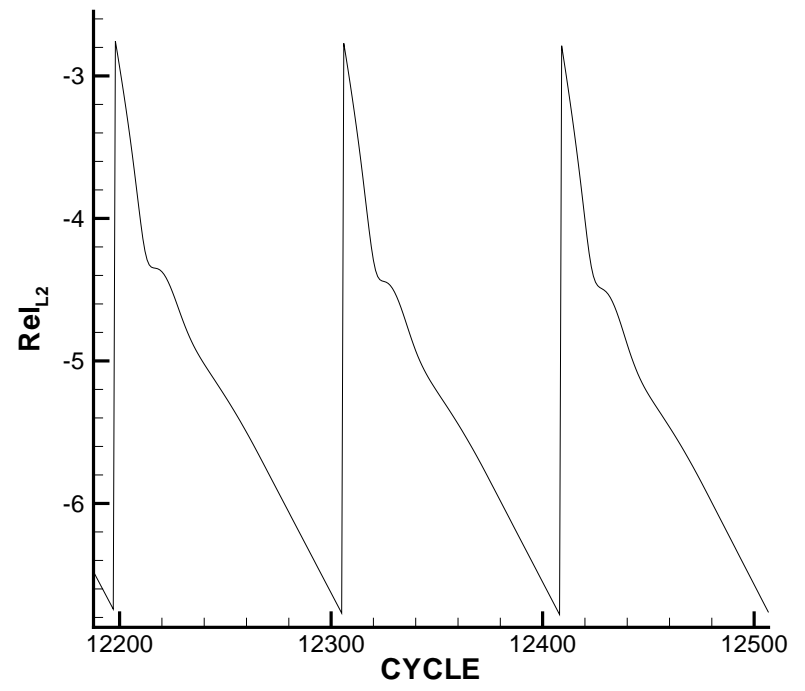

Fig. 15 Convergence for Block 3 of turbulent diffusion flame.

Fig. 14 Convergence for Block 2 of turbulent diffusion flame. 